\title{
Recent Developments of the Navier Stokes Multi Block (NSMB) CFD solver.
}

\author{
Y. Hoarau, D. Pena \\ ICUBE, Strasbourg University, FR-67000 Strasbourg, France \\ J.B. Vos $\ddagger$ D. Charbonnier, \\ CFS Engineering, EPFL Innovation Park, Batiment A, CH-1015 Lausanne, Switzerland \\ A. Gehri, \\ RUAG Aviation, Aerodynamics Department, CH-6032, Emmen, Switzerland \\ M. Braza, T. Deloze** \\ Institute de Mecanique des Fluides Toulouse (IMFT), Allee du Professeur Camille Soula, FR-31400 Toulouse, France \\ E. Laurendeau ${ }^{\dagger \dagger}$ \\ Ecole Polytechnique de Montréal, Montréal, Canada
}

\section{Introduction}

The Navier Stokes Multi Block solver NSMB was initially developed in 1992 at the Swiss Federal Institute of Technology (EPFL) in Lausanne, and from 1993 onwards in the NSMB consortium composed of different universities, research establishments and industries. Today NSMB is developed by IMF-Toulouse (IMFT), ICUBE (Strasbourg), University of Munchen (TUM, Germany), University of the Army in Munchen, Ecole Polytechnique Montreal, Airbus Defence and Space, RUAG Aviation and CFS Engineering.

At the Aerospace Sciences Meeting in 1998 an overview of the developments of NSMB was given ${ }^{1}$. Since then various papers have been published on NSMB, examples are ${ }^{2-9}$.

This paper will present several recent developments of NSMB and the use of NSMB for industrial test cases.

\section{The NSMB solver}

NSMB is a parallelized compressible Navier-Stokes Multi Block solver. NSMB includes a wide variety of numerical schemes, among them explicit (Runge-Kutta schemes) and implicit (LU-SGS) schemes for steady and unsteady simulations on fixed or moving grids (ALE, Re-meshing, Fluid Structure Interaction, ..). Space discretization schemes include $2^{\text {nd }}$ and $4^{\text {th }}$ order central schemes with artificial dissipation and several upwind schemes (Roe, AUSM, Van Leer ...) ranging from $1^{\text {st }}$ to $5^{\text {th }}$ order. Multigrid and full-multi grid are available

\footnotetext{
*Professor, hoarau@unistra.fr, AIAA member

${ }^{\dagger}$ PhD Student, dorian.pena@etu.unistra.fr

${ }^{\ddagger}$ Director, jan.vos@cfse.ch, Senior AIAA member

§Senior Scientist, dominique.charbonnier@cfse.ch

『Senior Scientist, alain.gehri@cfse.ch

॥ Professor, marianna.braze@imft.fr, AIAA member

** Scientist, deloze@gmail.com

${ }^{\dagger}$ Professor, eric.laurendeau@polymtl.ca, AIAA member
} 
to accelerate convergence to steady state. Dual time stepping is employed for unsteady simulations. Several pre-conditioning methods for low Mach number flows have been implemented, and for incompressible flows both the Artificial Compressibility method or a fully colocated SIMPLE scheme can be used. Many well validated turbulence models (0 to 5 equations) have been implemented, as well as Hybrid RANS-LES, LES models and a transition model. NSMB is also employed for hypersonic applications, and includes several levels of chemistry modeling for air, $\mathrm{CO} 2$ and N2, more details are given in this paper. To simplify the grid generation for complex geometries NSMB uses the so-called fully patch grid approach and it is not needed that grid lines are continous across block interfaces. A futher simplification of the grid generation for complex geometries and in particular for moving components is the chimera approach discussed in more detail in this paper. The next sections briefly summarize recent developments of NSMB.

\section{The Chimera method}

One of the important elements in CFD simulations is the spatial discretization. This is not a problem for a simple and static geometry, but it becomes a major issue when the geometry is complex and contains moving parts, and this is especially the case when using structured grids. In this case, the grid generation is the limiting factor for the simulations. To overcome this problem, several solutions have been proposed like the Immersed Boundary Method (IBM), Volume Of Fluid (VOF) or Chimera methods. The last one, chimera method (also called overset grid method) appears increasingly for complex research applications and more and more for large industrial applications.

The Chimera method was initially proposed by Benek et al. ${ }^{10}$ and the method was subsequently improved and employed for many cases $\left({ }^{11-22}\right)$. Its principle is to decompose the complex full domain into simple subdomains independently meshed by curvilinear grids. The only constraint is a superposition of grids. Besides simplifying the mesh generation, this technique offers also a powerful solution to manage moving bodies.

The chimera processus has been summarized for structured grids by Meakin. ${ }^{20}$ The process of chimera method can be divided into four main steps like described by Landmann ${ }^{23}$ (fig. 1):

- $1^{\text {st }}$ step : Detection of overlapped cells. This process consists in finding which cells are overlapped by other cells. The test is based only on the coordinates of the cells.

- $2^{\text {nd }}$ step : Determination of the overlapped cell status. Three kinds of overlapped cells exists ${ }^{23}$ :

a) Interpolated cells : the flow data are obtained by interpolation from other overlapping grids.

b) Calculated cells : the values come from the solution of the equations governing the flow.

c) Hole cells (or blanked/masked cells) : the values of this kind of cells are never used in the discretisation schemes or overlapped boundary conditions and they are not interpolated nor calculated.

- $3^{\text {rd }}$ step : Calculation of the interpolations parameters. In this step, the donor cells and the associated weights that compose the interpolation are evaluated for each valid overlapped cell.

- $4^{\text {th }}$ step : Calculation of the interpolation values.

A three-dimensional structured multi-block chimera method has been implemented in NSMB in order to simulate initially the free falling sphere in a tube. The developped chimera method is fully automatic with few, if not none, of user input and pre-processing and described in the following sub-sections.

\section{III.A. Detection of overlapped cells}

The search of overlapped cells is based on a test of inclusion on the coordinates. In order to accelerate the search, the mapping of coordinates in a virtual uniform Cartesian grid is performed. This method is well known as the bucket method. ${ }^{24-26}$ An inverse mapping algorithm creates an index array that link virtual grid to the real coordinates. The search begins on the virtual grid and it continues on the physical associated grid points. The creation of link array is time consuming but the gain in the search time is significant (at least a factor 20).

This algorithm is well known but the number of the virtual cells $\left(N_{v}\right)$ is a parameter not clearly defined. A simple test is performed in order to determinate the optimum value of $N_{v}$ in function of the number of real coordinates $N$. We consider two uniform three-dimensional Cartesian grids $\left(N_{x}=N_{y}=N_{z}=N=128\right)$ 

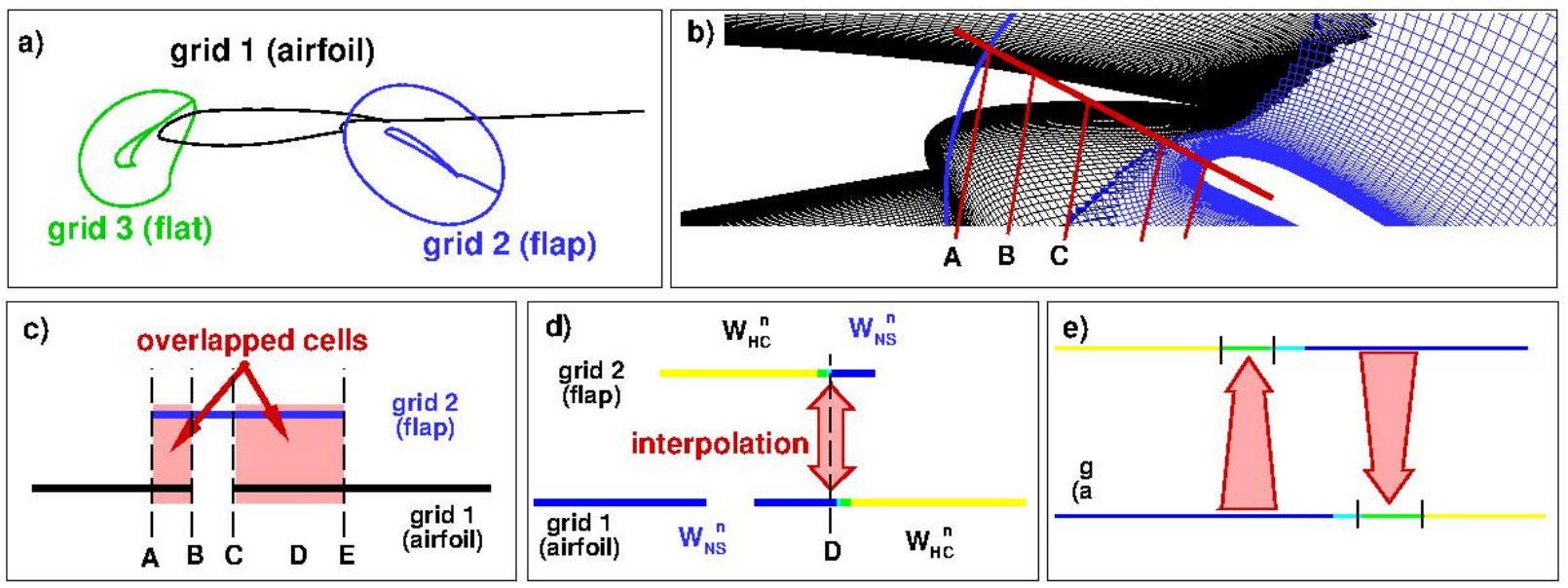

Figure 1: Main steps of the chimera method.

overlapping entire the cube $1 \times 1 \times 1$ domain. Identical results were obtained on a coarser and a finer grid. The cpu-time is determined for range $1 \leq N_{v} \leq 256$. In addition to the compromise between cpu-time decrease for virtual grid creation and cpu-time increase for searching the overset, the size required by the link array must be taken into account. The results, represented in figure 2 , show that the optimum value for the virtual cell number $N_{v}$ is the half of the number of physical coordinates $N$. The same test is performed for a grid with tangent hyperbolic distribution and, in this case, the optimum value correspond to $N$.

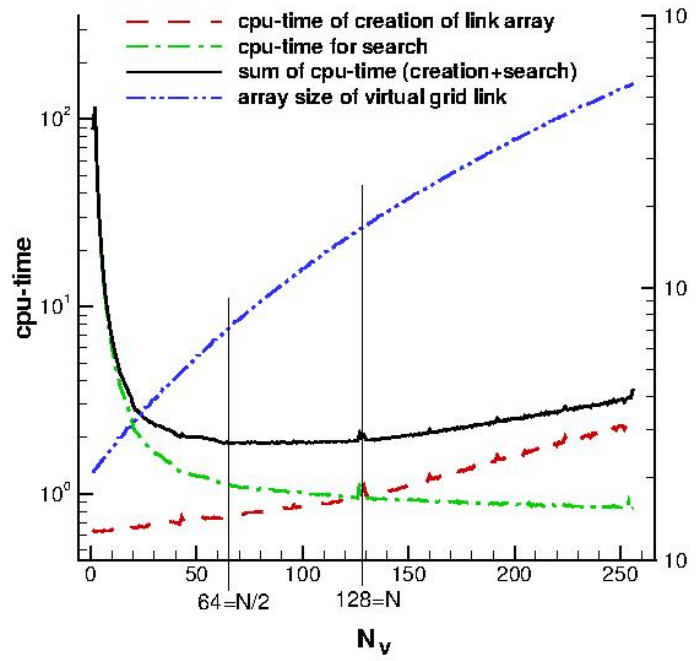

Figure 2: Representation of the cpu-time used for the indexation of real coordinates in virtual grid, cpu-time for searching overset and the size of the index between both grids versus the number of virtual box $N_{v}$ in one direction.

Another issue concerns the points that are inside a solid body. In this case, the cells are not detected as overlapped cells. To remove these cells, three methods are implemented. For simple cases (cylinder, sphere, channel) an analytical function is used. This solution is the quickest but the least flexible. For general cases, a second method is implemented using the closest wall. The dot product of the vector from the nearest wall cell center to cell center and the vector of the associated wall normal vector is calculated. If the dot product is negative the cell is in the solid region. This method requires the knowledge of the closest wall point and it is CPU time consumption. For the configuration of a sphere in a tube, having 6 millions of cells and 65000 wall cells, the second method is 30 times more expensive than the first one. A third method are implemented 
to combine the rapidity and the flexibility of the both methods. The solution take a simple way : if the issue is the absence of solid volume, the addition of solid discretisation solves the problem. The figure 3 illustrates the concept by representing the added solid mesh associated to the slap of a high lift profil (MDA 30P-30N). The solid mesh fits the fluid mesh to share the same wall distribution but it requires any precaution as skew or size ratio. The detection follows the same process than for a fluid overlap. To simplify the storage and the reading, the solid grids are in the same format than the fluid mesh (structured defiinition). This third approach of solid detection gathers simplicity, rapidity and accuracy.
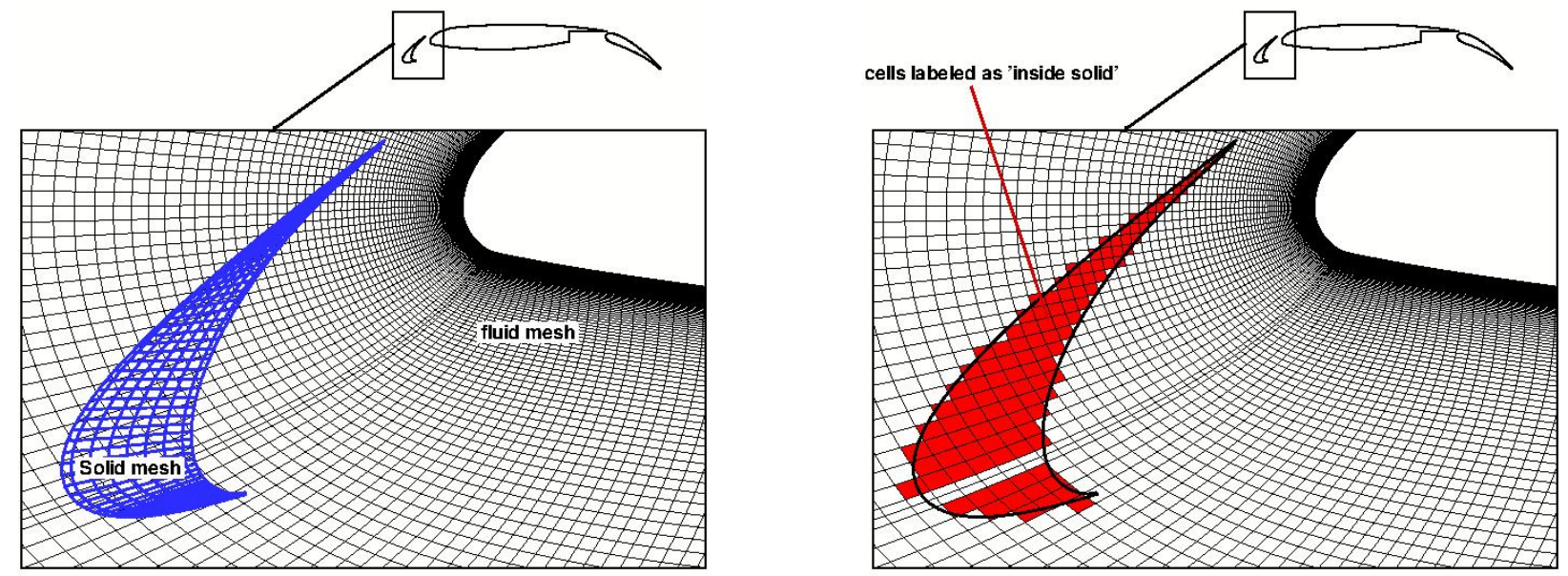

Figure 3: Scheme of the solid detection by added solid mesh for a high lift configuration, with the slat solid mesh and the fluid around the main profil (left) and the cells labeled as inside solid of the fluid mesh (right).

\section{III.B. Determination of the type of overlapped cells}

The second step of the chimera method is to define the type of the overlapped cells (calculated, interpolated, hole). In this step, among several overlapped cell, the best candidate to predict the flow state (calculated cell) and to give the flow solution to the other cells (interpolated cells) is determined. The criterion that justifies the choice is based on the best resolution of the physical problem. Four techniques are distinghuished, based on the criteria associated to the following quantities : user-defined grid ranking, user-defined cell quality, cell size and cell size in the wall-normal direction.

For a simple overset, the definition on an overlapped hierarchy of each block is enough for the cell type selection. All the cells of the highest ranking of the grid are defined as calculated cells. This solution is simple and fast but not suitable for complex overlapped layers where the definition of chimera grid ranking by the user is then needed. Based on the fact that the cell size is a criterion indicating the best discretization, Siikonen et al. ${ }^{26}$ or Liao et al. ${ }^{27}$ justify this choice by assigning the calculated cells type to the smallest ones. This criterion is locally adapted, automatic and requires no user input. A third criterion is added to the previous one and based on a user-defined cell quality. This way the user can force a specific kind of cell (calculated or interpolated) by associating to this cell a high quality value. This technique can force cell type by protecting or immunizing cells but the user input is binding and does not follow the grid movement. The cell size in the wall-normal direction was introduced by Landmann. ${ }^{23}$ The selection use the intersection segment between normal nearest wall and cell boundary. This technique is fully automatic and it is based on an important physical quality : the boundary layer. This test requires to search for the nearest wall and to calculate the intersection for each cell. In order to find a lighter, accurate and automatic criterion, we developed a similar criterion based on the distance of a cell to the nearest local wall. The nearest local wall is the wall in the same block where the cell is defined (figure 4 (left)). With this criterion, the overlapped cell with the smallest local near wall distance is calculated and the others are interpolated. This approach guarantees the resolution of Navier-Stokes equations in the nearest wall region and consequently each boundary layer is accurately computed. Moreover, this technique is based on the local wall distance and this quantity does not change with a moving grid (an update is however needed for deforming grids) 
whereas the criterion based on the wall normal intersection need an update after each grid movement.

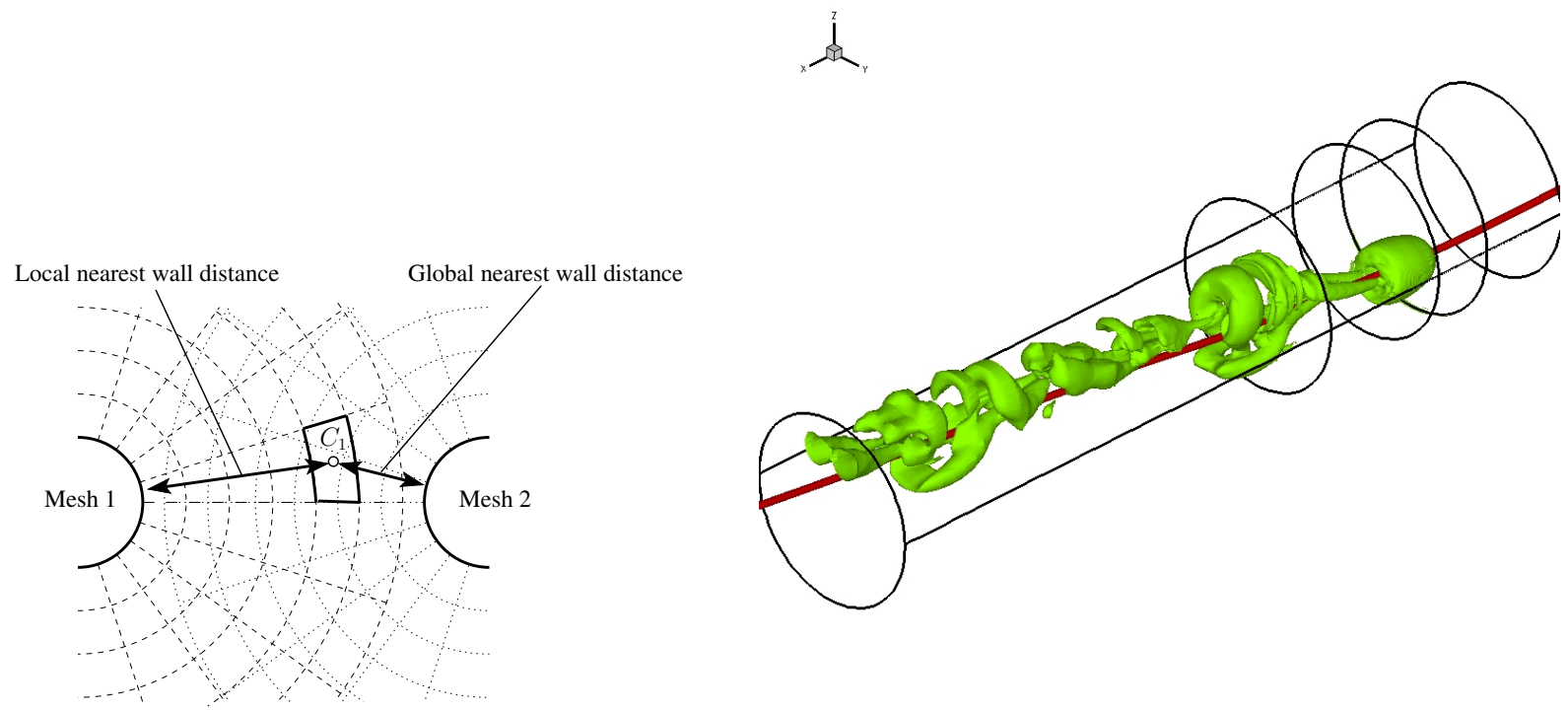

Figure 4: Left : Definition of local nearest wall distance and global nearest wall distance for the cell $C_{1}$ of the mesh 1 in the configuration of two overlapped polar meshes; Right : Flow structure of the free falling of a sphere in a tube.

In the case of the sphere in a circular tube, the criterion based on the cell size complicates the generation of the mesh. The same problem may be encountered in the configuration of a sphere near a plane wall. The cell aspect ratio of the near wall mesh is not the same as that of the spherical wall mesh and the cell volume is very difficult to control. In our case of a sphere in a tube with a cylindrical grid overlapped by a spherical grid, the criterion based on the volume does not give a proper solution. The new criterion based on the local near wall distance give the best answer. The figure 5 represents the visualization of the calculated cells in the 3 planes crossing the sphere center for the both cases.

\section{III.C. Buffer and hole cells}

The next step is the addition of a buffer layer. The buffer cells are in between interpolated and calculated cells. Their role is to move away the interpolation communications and thereby to avoid implicit interpolation. An implicit interpolation is an interpolation that uses an interpolated cell as a donor cell. The more the interpolation needs a large number of donor cells, the more the buffer layer needs to be wide. The importance of wide overset is admitted and described in several papers. ${ }^{28,29}$ The set-up of the buffer layer is implemented with an user-input. The user give a fixed number of layer and the algorithm changes the type of the cell from interpolated to calculated over this layer.

The last type of possible cells is the hole cell. This kind of cell is a non-necessary cell and it concerns interpolated cells. This cell does not appear in any discretization schemes. In order to save computation time, the interpolation process is avoided for this cell. After the buffer layers, a user-input imposes a number of interpolated cells in the hole region. These new cells are considered as ghost-cells. The minimum number of ghost-cells is two because the numerical schemes used in NSMB require two neighbor cells (for most simulations a fourth order central scheme with artificial dissipation was used).

\section{III.D. The interpolation}

The interpolation is a key element of the chimera method. It allows the passage of flow information between overlapped grids and it creates the link. The influence of interpolation has already been investigated in several studies (Delfs, ${ }^{30}$ Sherer \& Scott ${ }^{31}$ and Sengupta et al. ${ }^{32}$ ). The simplest treatment of an interface consists in the interpolation of all state variables. ${ }^{10,15,33-35}$ However, this approach does not guarantee the global mass conservation as explained by Wang et al. ${ }^{36}$ Some studies found a solution to ensure the global conservation for compressible flows ${ }^{37}$ or for incompressible flows (Wright and Shyy ${ }^{38}$ or Tang et al. ${ }^{39}$ ) with a correction of interpolated values. The accuracy of interpolation is important and different schemes of interpolation are 

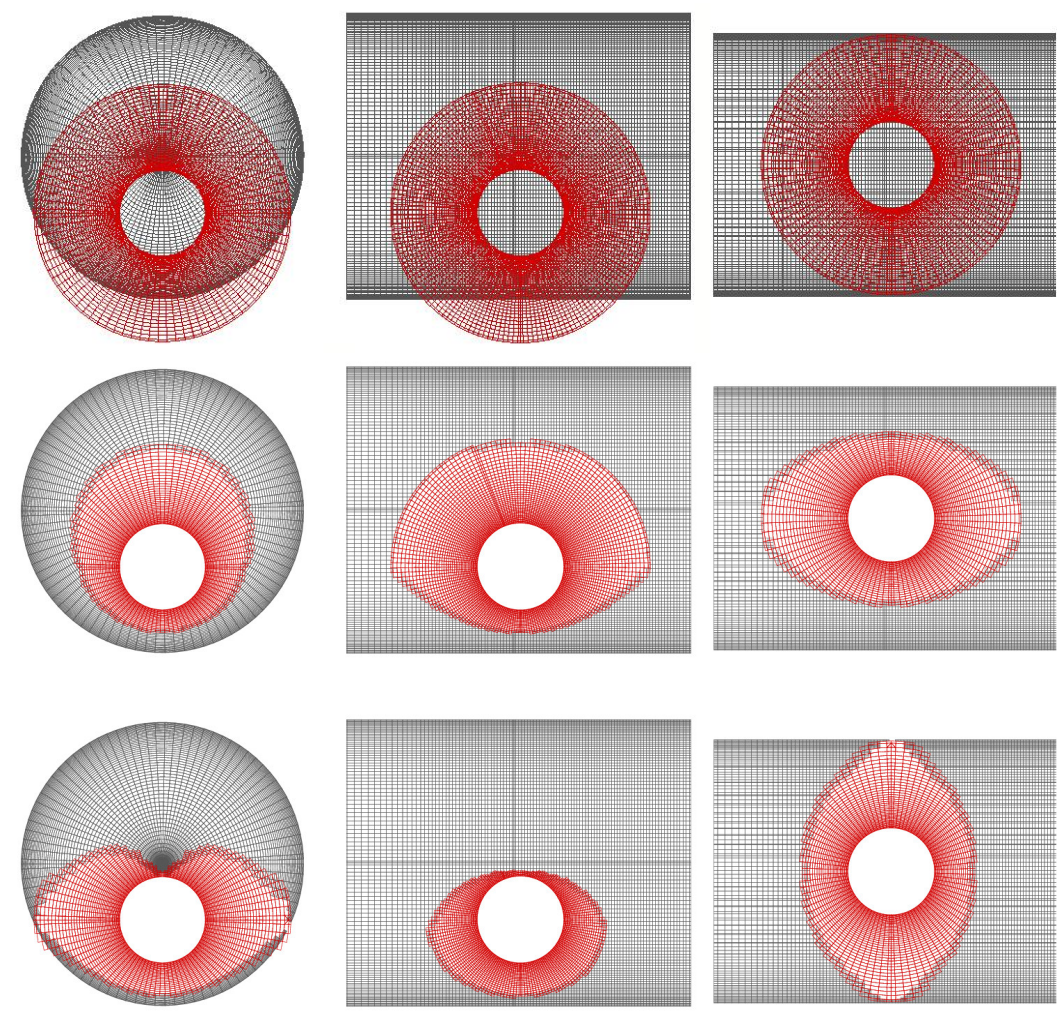

Figure 5: Visualization of the calculated cells for the chimera cell selection before cell selection ( $1^{\text {st }}$ row), using cell volume criterion ( $2^{\text {nd }}$ row) and using the nearest local wall distance criterion $\left(3^{r d}\right.$ row) for the cross section of the tube ( $1^{\text {st }}$ line), the streamwise plane in the symmetric plane ( $2^{\text {nd }}$ line) and streamwise plane perpendicular to the symmetric plane $\left(3^{\text {rd }}\right.$ line $)$

used : interpolation based on Lagrange polynomials, ${ }^{29,31,32,40,41}$ trilinear interpolation, ${ }^{39,42-44}$ quadratic interpolation $^{45}$ or tetravolumic interpolation. ${ }^{26,46}$

The interpolation scheme depends on the physical problem and the overset width. A highly-accurate interpolation needs many donor cells and it requires a wide overset. In our case of the sphere in a circular pipe, the gap between the sphere wall and tube wall does not provide a wide overset nor a large stencil of donor cells. For this reason, we chose some interpolation that use a low number of donor cells. The choice focus itself on the trilinear, tetravolumic and weighted inverse distance interpolations.

The inverse distance weighted interpolation is based on the distance between the points. The implementation is simple and its advantage is the flexibility of the stencil of points. The formula used is given by the following relation :

$$
f_{M}=\frac{\sum_{i=1}^{N} \frac{f_{i}}{d_{i}^{\alpha}}}{\sum_{i=1}^{N} \frac{1}{d_{i}^{\alpha}}}
$$

where $N$ is the number of the stencil points, $f_{i}$ the value at the point $i, \alpha$ is the weighting exponent (in our case equal to 2) and $d_{i}$ is the distance from the stencil point $i$ to the interpolation point $M$.

The second interpolation is the tetravolumic interpolation based on the tetrahedral volumes composed between the four nearest cells. The interpolation weights are given by the opposite tetrahedral volume :

$$
\begin{gathered}
f_{M}=\sum_{i=A, B, C} W_{i} f_{i} \\
\text { with } W_{A}=\frac{V_{B C M}}{V_{A B C}}, W_{B}=\frac{V_{A C M}}{V_{A B C}} \text { and } W_{C}=\frac{V_{A B M}}{V_{A B C}}
\end{gathered}
$$

with $W$ the weight of the interpolation and $V_{A C M}$ the volume of the triangle $(A B C)$. In three dimensions, the triangle volume is replaced by the tetrahedral volume. 
The third interpolation is the trilinear one. We use a trilinear interpolation based on a linear interpolation on each direction. In two-dimensional example of the figure 6 , the first linear interpolation yields the values $f_{Q}$ and $f_{P}$ then a second linear interpolation provides $f_{M}$. In three-dimensions, three steps of linear interpolation are required.

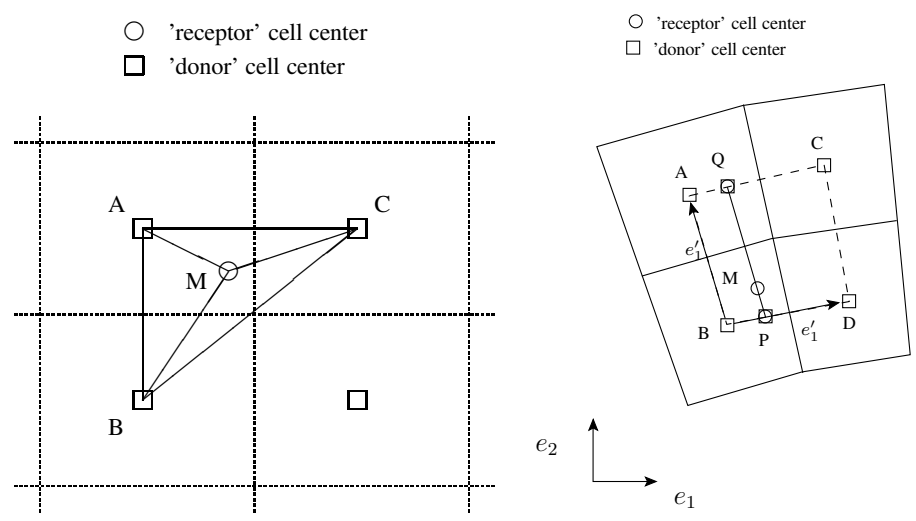

Figure 6: Tetra-volumic (left) and tri-linear interpolation (right) interpolation schemes.

The developed algorithms were inserted in the École Polytechnique de Montréal 2D RANS code NSCODE. Verification of the interpolation schemes were made in Pigeon ${ }^{47}$ and the methods robustness was shown for a multi-element airfoil slat/gap optimization problem. ${ }^{48}$

\section{III.E. Application of the chimera method}

The method described in the previous paragraph was validated using three different test cases : the simulation of flow around a sphere in an infinty domain, a flow around a sphere translating uniformly along a flat wall, and finally a sphere falling freely in a tube filled with a Newtonian fluid at rest. With each test case the complexity rises, so one part of a single flow, then an interaction between two walls defined by two independent chimera meshes, and finally the case of an interaction fluid / solid of a free falling body represented by on overset grid. These test cases were also selected for the availability of numerical or/and experimental results for comparison.

\section{III.E.1. Flow past a sphere}

The flow around a sphere is the topic of large number of experimental studies and numerical studies. ${ }^{49-55}$ The flow state is well known and clearly defined by a single parameter, the Reynolds number $(R e=U d / \nu$, with $U$ the freestream velocity, $d$ the diameter of the sphere and $\nu$ the kinetic viscosity). For low Reynolds number $(R e<212)$ the flow is steady and axisymmetric, then for $R e>212$ the axisymmetry is broken into a steady non-axisymmetric flow and the Hopf bifurcation appears at $R e=273$ when the flow becomes unsteady with horse-shoe vortex shedding.

This case test is the first step of the validation and it demonstrates the ability and the accuracy of the chimera method for a simple three dimensional computation largely referenced. The simulations are performed by two set of grids : an chimera grid composed of a cartesian background grid overlapped by a spherical classical body-fitted mesh.

The representation of the isolines of streamwise velocity in the streamwise plane (figure 7 ) shows the wake behind the sphere with a recirculation. The shape of the vortex is a torus like described in the literature (Johnson et al. ${ }^{53}$ for example). The dashed line on the figure 7 represents the external border of the spherical grid. The flow information crosses correctly this overset border.

The comparison of the results with results from literature is based on three physical quantities : the drag coefficient $\left(C_{D}\right)$, the recirculation length $(L)$ and the separation angle $(\theta)$. For this range of Reynolds numbers, the flow is axisymmetric and the lift force is zero. Comparing the drag coefficients with the results of Bouchet et al. ${ }^{52}$ shows a good agreement, differences do not exceed $0.52 \%$ and this demonstrates the good resolution of the overlapped grids. The shape descriptions $(L$ and $\theta)$ are in good agreement with the literature results and are shown in Fig. 8. The results are compared with datas obtained by experimental 

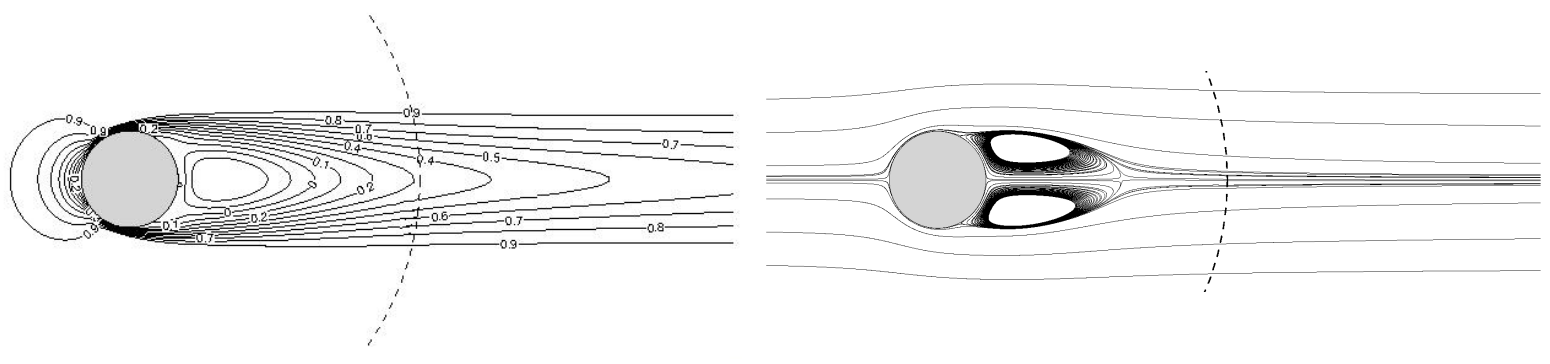

Figure 7: Isovalues of the streamwise velocity component (left) and the streamlines (right).

studies (Taneda, ${ }^{49}$ Nakamura ${ }^{56}$ and Roos \& Willmarth ${ }^{57}$ ) and by numerical studies (Tomboulides et al., ${ }^{55}$ Mittal, ${ }^{58}$ Bagchi \& Balachandar ${ }^{59}$ and Bouchet et al. $\left.{ }^{52}\right)$.
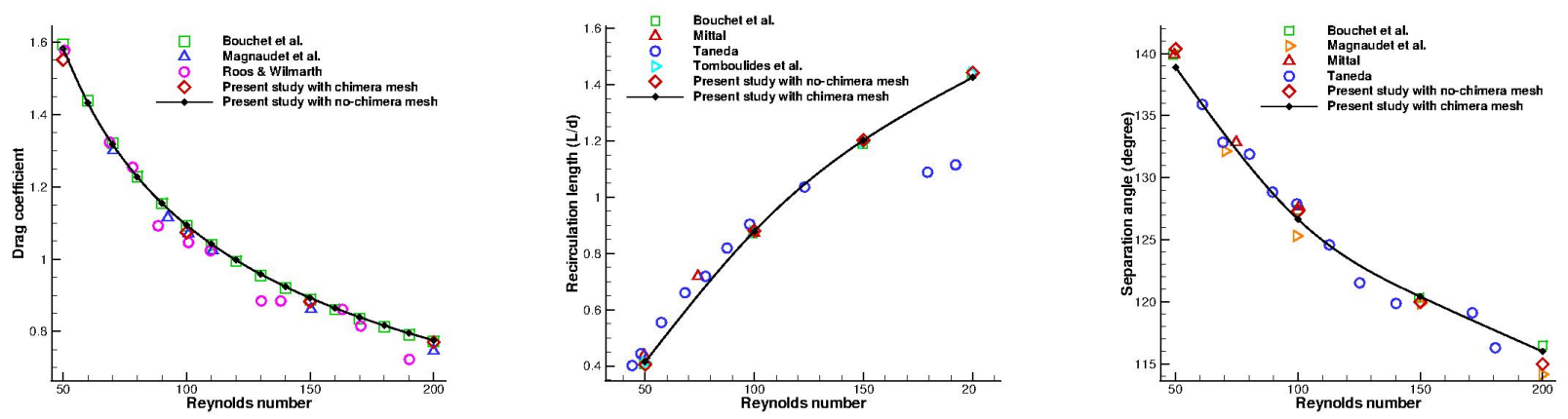

Figure 8: Drag coefficient $C_{D}$, recirculation length $(L / d)$ and separated angle versus Reynolds number for the flow past a sphere and $50 \leq R e \leq 200$.

\section{III.E.2. Flow around a sphere translating along a plane wall}

To complete the validation of the three-dimensional chimera method, we consider a configuration with an interaction between two walls yielding a border of overlapping. We simulate the flow past a sphere in a uniform translation parallel to a plane wall. This configuration has been previously simulated by Zeng et al. ${ }^{60}$ The parameters for this configuration are the distance between the sphere center and the wall $(L / d)$ and the Reynolds number $R e=U . d / \nu$, with $U$ the velocity of the sphere translation and $\nu$ the kinematic viscosity of the fluid. The geometry is meshed with a Cartesian grid, which is refined along the plane wall, and a spherical grid which is refined near the sphere wall. The criterion for chimera cell selection is based on the definition of a boundary layer for the background grid where the cells are always calculated. Elsewhere the cells of spherical mesh are calculated. The motion of the sphere is implemented with the wall condition of the plate and using an inlet boundary condition. The mesh for parallel simulations is decomposed into 32 sub-blocks. The range of the Reynolds number is $10 \leq R e \leq 250$ and the studied distance is $L / d=1$ and $L / d=0.75$.

The presence of the plane wall breaks the axisymmetric geometry and for all Reynolds numbers a lift force exists. Two mechanisms are the source of this force. The first is linked to the strong interaction between the wall and the wake of the sphere. Figure 9 shows the isovalues of the streamwise velocity in the wall normal plane for the case $R e=200$ and $L / d=1.00$. The distribution of the flow shows that the up/top asymmetric has been lossed, resulting in a lift force directed away from the wall. The second mechanism is linked to the high velocity in the gap associated to a low pressure. This behavior gives a force with opposite direction (attractive force) to the first mechanism. The resultant force tends to push the sphere away from the wall. The streamlines plotted along the streamwise wall normal plane (figure 10) represents the vortex structure for $R e=200$ and $L / d=0.75$. The same case performed by Zeng et al. ${ }^{60}$ is plotted below our results for comparison (Fig. 10). The overset border is not visible in the results, proving that the chimera 
implementation is correct.

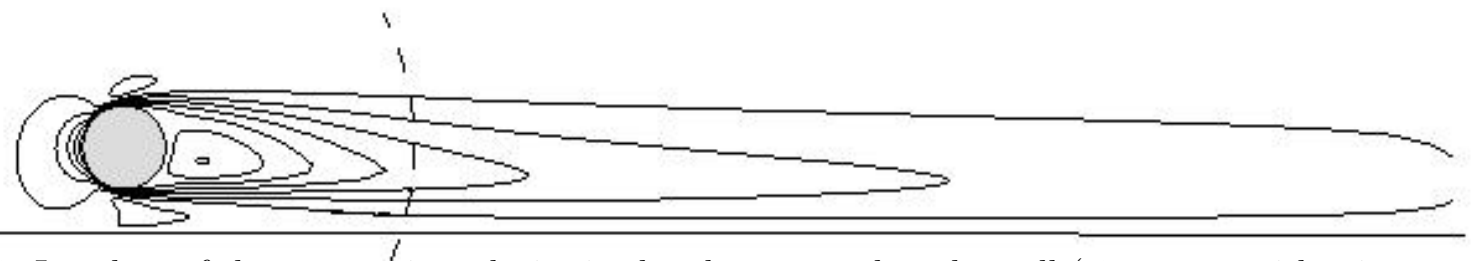

Figure 9: Isovalues of the streamwise velocity in the plane normal to the wall (-0.3 to 1.1 with a increment of 0.2 )

The results of the aerodynamic coefficients are in close agreement with those reported by Zeng et al, ${ }^{60}$ differences are below 1\%, see Fig. 10.

a)

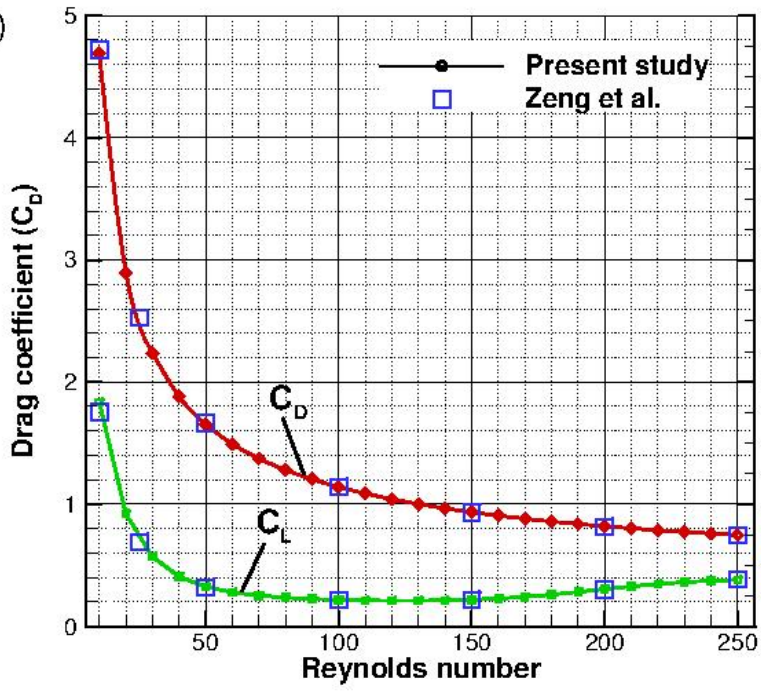

b)

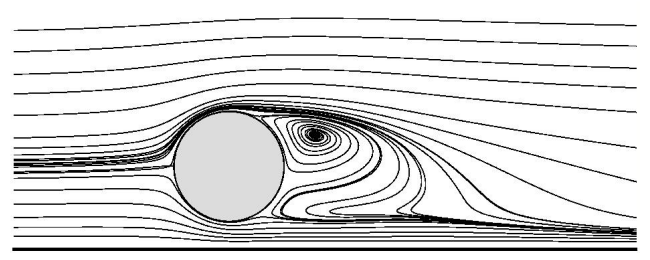

c)

Zeng et al.

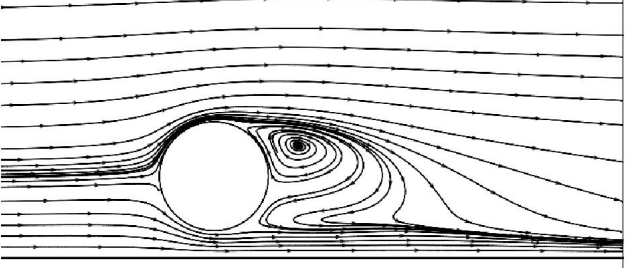

Figure 10: The drag and lift coefficient for $\mathrm{L} / \mathrm{d}=1.00$ and versus Reynolds numbers and b,c) streamlines in the plane normal to the wall for the case $R e=200, L / d=0.75$ for the present study (left) and extract to Zeng et al. ${ }^{60}$ (right)

\section{III.E.3. Falling bodies}

Examples of the use of the Chimera method is the study of the transition to chaos of a sphere falling into a tube and the apex cover separation process for the Advanced Re-entry Vehicle (ARV). Deloze et al ${ }^{8}$ studied the transition scenario of the freely falling sphere confined in a tube. The primary bifurcation lies between $G=150$ and 160 ( $G$ being the Galileo number defined as $G=\frac{\sqrt{\left|\rho_{s} / \rho-1\right| g d^{3}}}{\nu}$. The axisymmetry breaking arising in the wake generates a steady lift and the sphere is also exposed to the repulsive wakewall interaction. No stable state corresponding to static equilibrium of the lift and repulsion from the wall were found. Instead, the sphere tends to oscillate in a plane before switching to a helical trajectory. The latter presents the counterpart of the steady oblique trajectory of an unconfined sphere. The unconfined sphere undergoes a secondary instability at about $\mathrm{G}=190$. This instability leads to an unsteady periodic regime and to a chaotic trajectory (figure 4(right)).

The ARV was an ESA funded study for a both a cargo and crew transportation system to service the International Space Station (ISS). The project was abandoned in 2012. The ARV geometry is a blunt axisymmetrical capsule similar to the ARD (Advanced Re-entry Demonstrator) flown by ESA in 1998. The 
ARV-APEX cover is extracted from the module by two drogue parachutes that are modelled through forces acting on the APEX cover. The unsteady 6DOF CFD simulations were made using the chimera method on three different grids:

- a back ground mesh that is fixed in time;

- a mesh around the ARV capsule that moves in time following a prescribed initial velocity;

- a mesh around the ARV cover that moves in time using the the prescribed forces from the two drogue parachutes together with the aerodynamic forces acting on the cover.

Figure 11 shows a picture of these three chimera grids. The main question to be answered in these simulation was whether the separation process could lead to dynamic instabilities. Figure 12 shows the velocity field around the ARV and the APEX cover at four different instances in time, and one can observe the movement of the APEX cover due to the wake of the capsule.

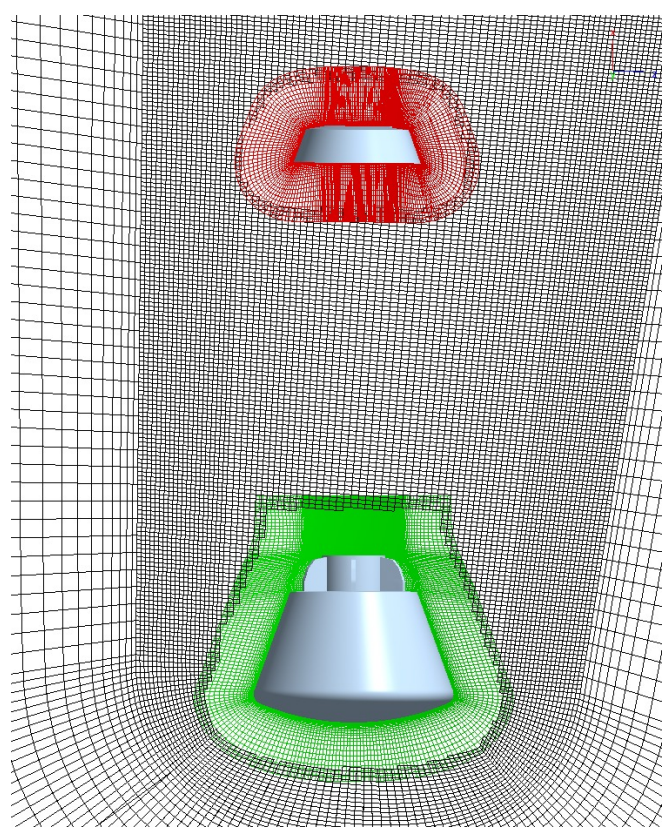

Figure 11: Chimera grid used in the ARV APEX cover separation simulations.

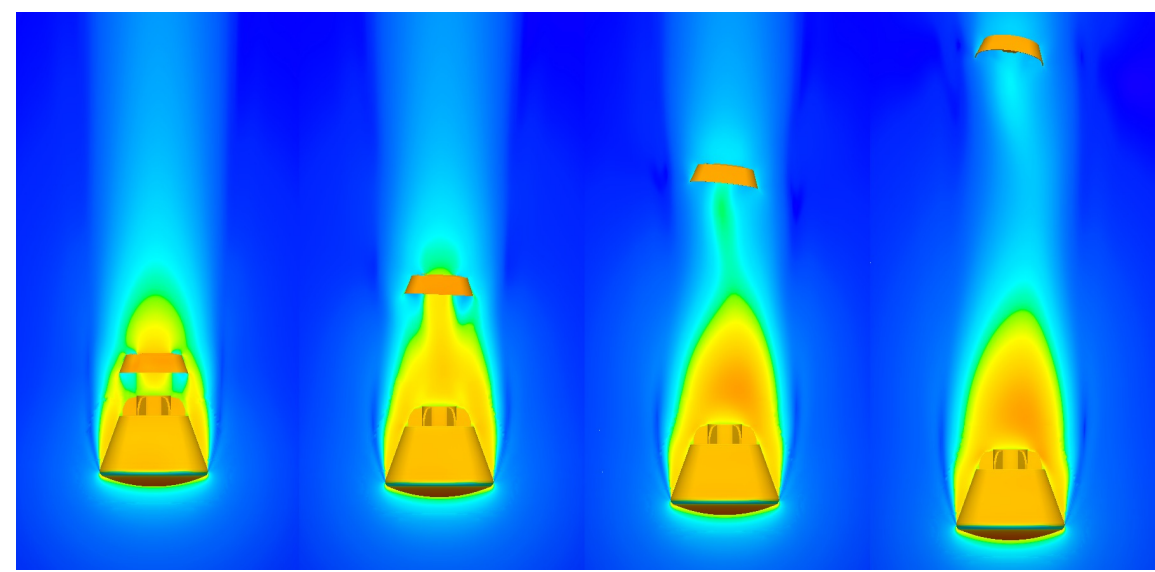

Figure 12: Velocity field around the ARV and APEX cover at $t=0.2,0.4,0.6$ and 0.8 secs. 


\section{Turbulence modelling}

\section{IV.A. Current modelling in NSMB}

The NSMB solver contains a large variety of turbulence modelling closures including LES, RANS, URANS and hybrid RANS-LES approaches. In the context of RANS-URANS, NSMB includes standard one-equation modelling as for example the Spalart-Allmaras ${ }^{61}$ and its variants for improved near-wall treatment (Edwards and Chandra ${ }^{62}$ ) and compressibility corrections of Secundov (Shur et al ${ }^{63}$, Spalart ${ }^{64}$ ), two-equation modelling including the $k-\varepsilon$ and its various low-Reynolds number versions (example: the Chien modelling ${ }^{65}$ ), the $k-\omega$ modelling of Wilcox ${ }^{66}$ and the $k-\omega-S S T^{67}$, as well as non-linear models among which the Abe, Jang and Leschziner ${ }^{68}$ and the DRSM (Differential Reynolds Stress Modelling) of Launder, Reece anf Rodi $^{69}$ and its low-Reynolds number version by Shima et al $^{70}$, as well as the SSG (Speziale, Gatski, Sarkar) modelling ${ }^{71}$. A specific attention has been paid in order to better capture non-equilibrium turbulence effects in high Reynolds number flows around bodies, where LES approaches are quite costly. NSMB involves the SAS-Scale Adaptive Simulation ${ }^{72}$ and the OES - Organized Eddy Simulation modelling ${ }^{73-75}$ including a tensorial eddy-viscosity concept derived from DRSM and able to account for near-wall turbulence stress anisotropy. OES modelling is particularly efficient in predicting the dynamics of unsteady coherent structures developing from the near-wall region and convected in the wake and ensures an improved prediction of the aerodynamic/hydrodynamic forces and of their fluctuations. Moreover, NSMB includes various hybrid RANS-LES methods, in order to take benefit from advanced URANS in the near-region and of LES in the outer region characterized by convected vortex dynamics farther from the wall. In this context, the DES (Detached Eddy Simulation ${ }^{76}$ ), DDES (Delayed Detached Eddy Simulation ${ }^{77}$ ) and IDDES (Improved Delayed Detached Eddy Simulation ${ }^{78}$ ) approaches have been implemented. An example of DDES around a supercritical wing using NSMB can be found in the AIAA paper by Grossi et al ${ }^{79}$, in the context of the European research program ATAAC, Advanced Turbulence simulations for aerodynamic application challenges, http://cfd.mace.manchester.ac.uk/twiki/bin/view/ATAAC/WebHome. A considerable improvement of the detached eddy simulation has been accomplished within NSMB by implementing the OES modelling in the URANS part of the DES and DDES in case of fixed and moving bodies including fluid-structure interaction $^{7,80}$. The DDES-OES modelling has been coupled with the ALE - Arbitrary Lagrangian-Eulerian Method $^{81}$ accounting for mesh movement and deformation during the fluid-structure interaction process for moving/deforming configurations. The DDES-OES modelling has been also successfully applied in the simulation of the transonic buffet around supercritical wings in collaboration with Dassault Aviation in the context of the TFAST European research program, Transition location effect on shock wave boundary layer interaction, http://tfast.eu/, (Szubert et $\mathrm{al}^{82}$ ), where in addition, the transition location effect upstream of the shock-boundary layer interaction has been studied and an optimisation of its position has been achieved by means of kriging method. These recent developments have been able to capture the fluid-elastic instability leading to flutter in a cylinders bundle at high Reynolds number, (Shinde et al, ${ }^{7}$ figures ?? and 13 left) as well as to predict the forces fluctuations around a wing of type Airbus A3xx in the context of the Smart Morphing Center activities, www.smartwing.org, in collaboration with Airbus - Emerging technologies and Concepts, Zilli, 2015. ${ }^{83}$

Among recent developments in turbulence modelling available in NSMB, a considerable effort has been paid in the development of stochastic forcing in the context of upscale turbulence modelling in order to enhance an eddy-blocking effect between the separated shear layers, by means of re-injection of turbulence from higher-order POD (Proper Orthogonal Decomposition) modes (Szubert et al ${ }^{84}$ ) - the IOES (Improved OES) approach. This method, inspired from inverse turbulence cascade and upscale turbulence modelling (Hunt et $\mathrm{al}^{85}$ ) produces a thinning of the shear layers and a reduction of the wakes width with considerable improvement of the wall pressure prediction and of the aerodynamic forces (Szubert et $\mathrm{al}^{84}$ ). The OES method has been successfully applied in the case of the Dassault Aviation V2C wing (TFAST European program), in comparison with the DDES method (Szubert et $\mathrm{al}^{82}$ ), where a considerable improvement of the forces and suction effect has been achieved. A view of the POD modes used for the stochastic forcing in the $\mathrm{V} 2 \mathrm{C}$ transonic buffet case are presented in the figure 14.

Moreover, a specific attention has been paid in implementing transition modelling in NSMB, in the context of the PhD thesis of Damien Szubert (Szubert, 2015 ${ }^{86}$ ). The $\gamma-R_{\theta}$ transition modelling of Langtry and Menter ${ }^{87}$ has been implemented and led to simulation of transonic buffet around the V2C - 3D configuration within the TFAST project (figure 13 right). 


\section{IV.B. Simulations of turbulent flows}

The following results concern the recent developments in turbulence modelling within NSMB. The DDESOES modelling has been employed in a tandem of cylinders configuration for the prediction of the aerodynamic forces, predominant frequencies associated with landing gear noise, as well as the MIV - Movement Induced Vibration developing by the downstream cylinder due to the shearing mechanism created by the upstream cylinder at Reynolds numbers in the range 100000 - 170 000. An analysis of the flow dynamics for the static configuration can be found in an experimental study of Elhimer et al ${ }^{88}$ by means of Time-Resolved PIV carried out in the S4 wind tunnel of IMFT.

The mesh used is of $12 \mathrm{M}$ and was provided by NTS - St Petersbourg in the context of the ATAAC EUprogram. Figure 15 shows instantaneous vorticity fields colored by pressure, at organized large wavelength. The spectra in figure 16 show the development of the von Kármán mode and of the Kelvin-Helmholtz vortices mode in comparison with experiments by Jenkins et al ${ }^{89}$. Figure 16 (left) shows the turbulence structure developed after free vertical vibration (in the direction of lift force), spontaneously developed by the downstream cylinder allowed for one-degree-of-freedom vibration, when the reduced velocity $\left(u^{*}=\right.$ $\left.U_{\text {inf }} /\left(f_{0} D\right)\right)$ becomes higher than $3, U_{\text {inf }}$ being the upstream velocity and f0 the structural eigenfrequency. The simulations have been carried out for a Scruton number (mass-damping) of 1. Figure 16 (right) shows the progressive amplification towards negative damping (i.e. flutter) of the downstream cylinder's motion, as the reduced velocity increases. This evolution also shows the progressive increase of the phase-lag as the fluid-structure interaction regime leaves out the VIV (Vortex Induced Vibration and enters to MIV (Movement Induced Vibration) behaviour for reduced velocities higher than 3.
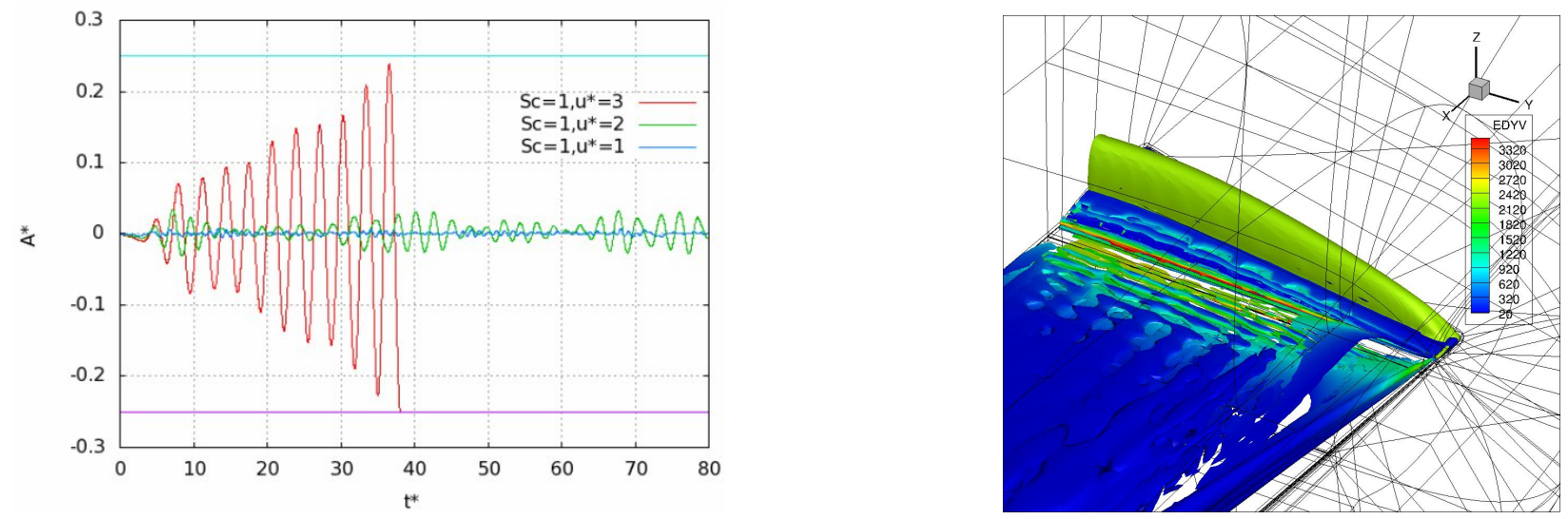

Figure 13: Left : Development of vibratory instability and flutter as the reduced velocity increases; Right $: \lambda_{2}$ criteria colored by $\mu_{t} / \mu$ of the flow around the Dassault Aviation V2C 3D wing, $M=0.70$, Reynolds around $4.10^{6}$ and $\alpha=7^{\circ}$.

Figure 18 shows the prediction of oblique choc reflexion over a boundary layer, according to the TFAST test-case measured by Technical University of Delft. The mesh is of $30 \mathrm{M}$ points and a first overview of the computations can be found in the CTR Biannual report of 2014 by Szubert et $\mathrm{al}^{90}$ in collaboration with the group of Prof. P. Moin.

Mode 80 - u

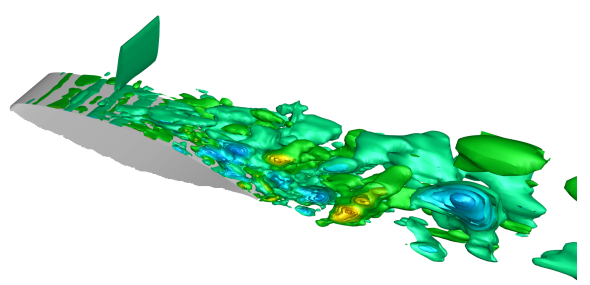

Mode $72-$ w

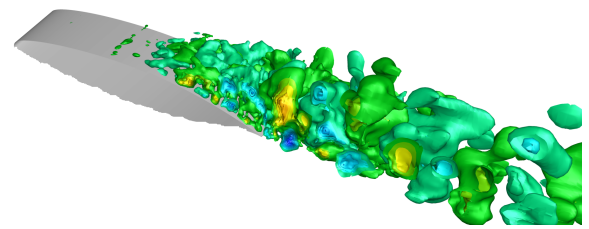

Figure 14: POD reconstructed instantaneous turbulent velocity fields of the flow around the Dassault Aviation V2C aifoil, $M=0.70, R e=3.24510^{6}$ and $\alpha=6^{\circ}$, DDES-k $\omega$ SST. 

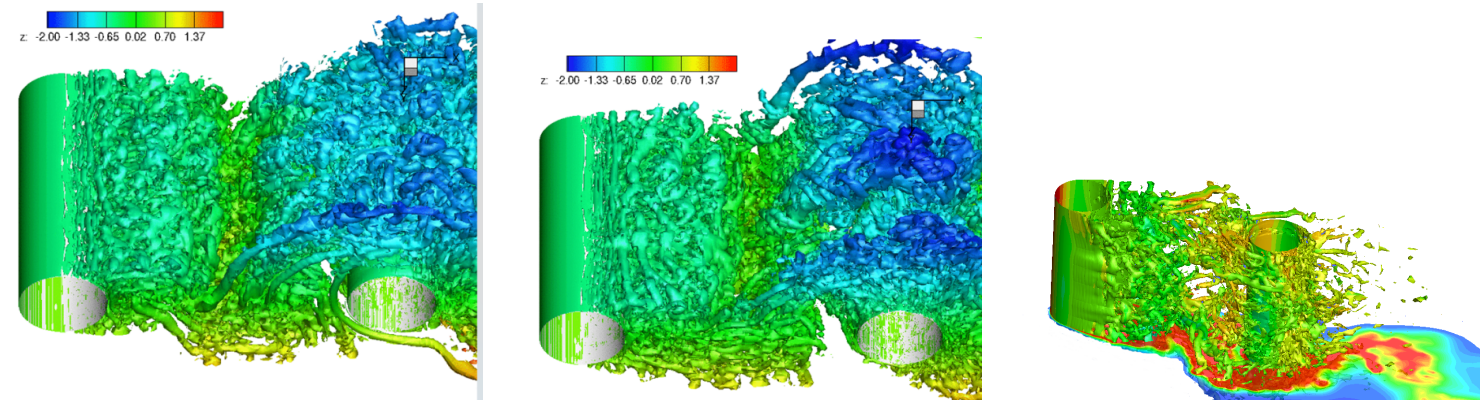

Figure 15: Simulation of the turbulent flow around a tandem of cylinders at Reynolds number 160,000 and centers distance 3.75 D. Illustration of turbulent motion development and of streamwise vorticity filaments formation (blue structures) arranged spanwisely periodic, according to a large-scale wavelength, (Test-case of the ATAAC European program).
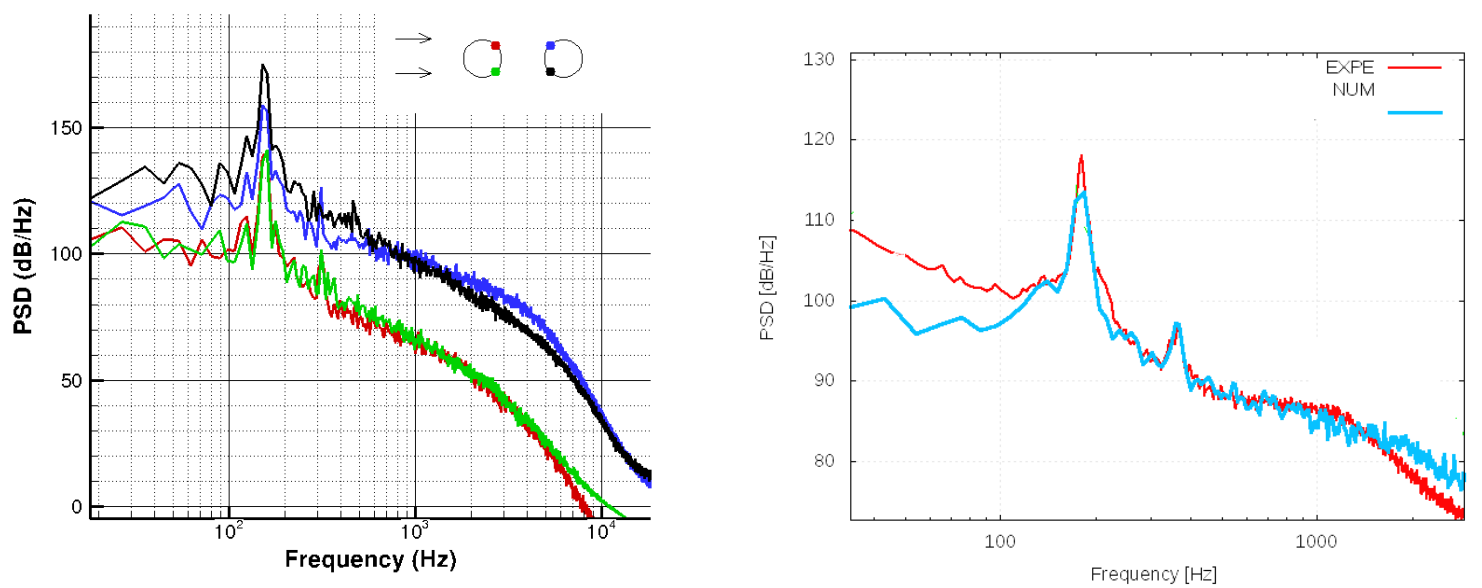

Figure 16: Power Spectral Density of the wall pressure at four points located at $\theta= \pm 45^{\circ}$ on the cylinders surface and comparison of normalised spectral density with experimens by Jenkins et al ${ }^{89}$.

\section{Icing modelling}

In-flight aircraft icing is a main research area within the aeronautic community. Ice accretion mostly results in severe aerodynamic degradation and is the major cause of flight incidents. Because of the rigorous certification process and since all configurations cannot be experimentally tested, aircraft in-flight icing must be simulated via computational fluid dynamics.

By impacting on aircraft structures, the supercooled water droplets carried by air can immediately freeze upon impact or flow as a water film depending on the surface temperature. Two main types of ice known as rime ice and glaze ice are formed depending on the environmental conditions.

The dry rime ice usually forms at low flow speeds and low flow temperatures when the entire droplet freezes at its impact location. Higher flow temperatures and flow speeds induce the formation of wet glaze ice. In that situation a fraction of the droplet freezes at impact point and the other portion flows as a thin water film runback and freezes downstream. The modification of the geometry due to icing results in large aerodynamic degradation.

Ice accretion codes used by industries are commonly based on a 2D inviscid panel method for the flow simulation, a boundary layer method based on empirical relations for the heat transfer coefficient evaluation, a Lagrangian formulation to simulate the droplet trajectories and the so-called Messinger model to predict the ice thickness. A better accuracy can be obtained by repeating this one-way coupling process until the 

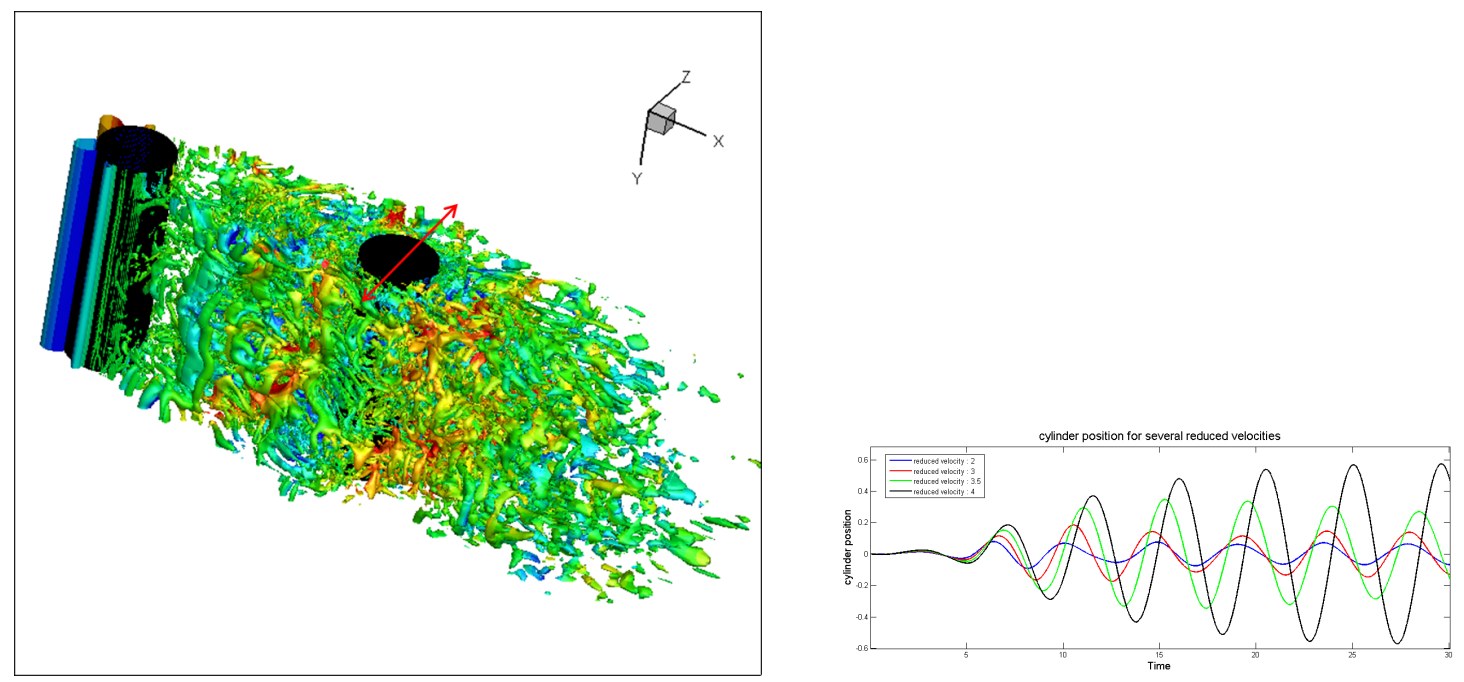

Figure 17: Evolution of the downstream cylinders position as a function of time for increasing reduced velocity and Scruton number of 1. Progressive appearance of phase-lag characterizing the MIV behaviour and negative damping.
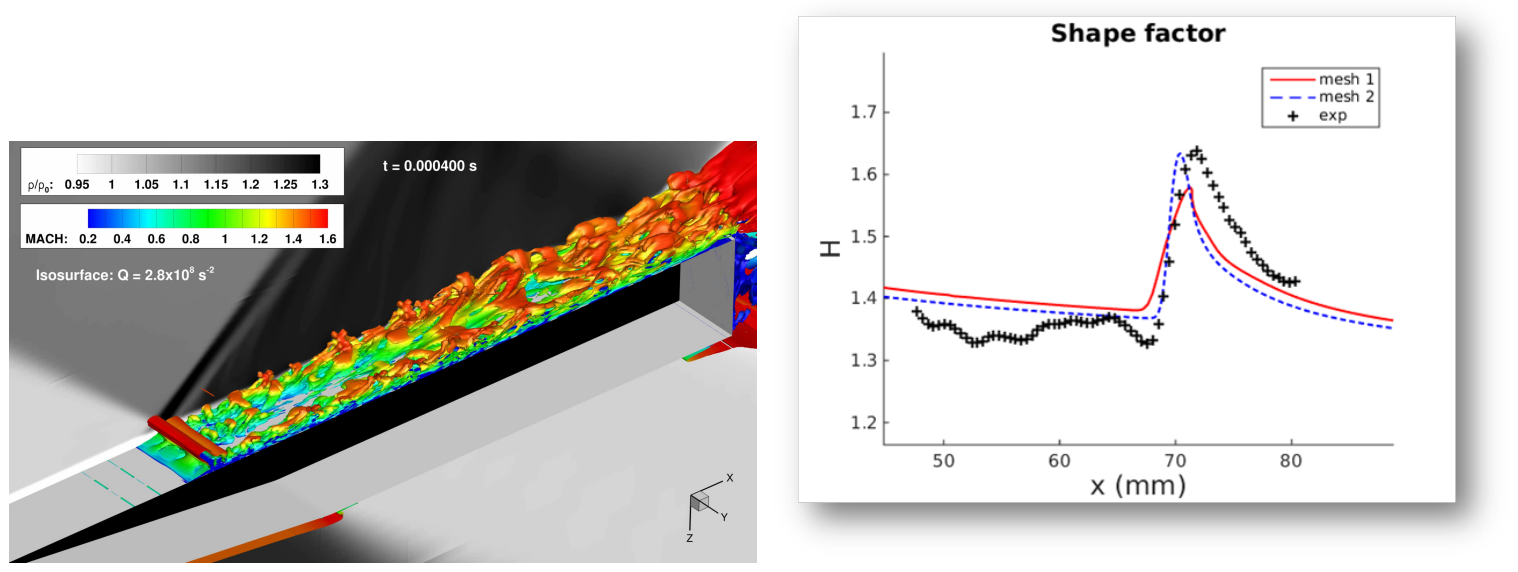

Figure 18: Oblique shock interaction, upstream Mach number 1.7 and Reynolds number of $36 \mathrm{M}$. IDDES and DDES computations using the Spalart-Allmaras model. Comparison of the boundary layer shape factor with PIV experiments of the Technical University of Delft (R. Giepman, F. Schrijer and B. van Oudheusden).

desired exposure time (multi-step approach). ${ }^{91}$

In NSMB, the supercooled water droplets field and impingement locations are obtained by means of an Eulerian approach similarly to FENSAP-ICE ${ }^{92}$ and ONICE3D ${ }^{93}$ The ice thickness is calculated by resolving the Shallow Water Icing Model (SWIM) for three-dimensional and multi-block ice accretion simulations. ${ }^{92}$ A Mesh deformation is used to track the ice/air interface through time for multi-steps calculations. These approaches allows a seamless integration to Navier-Stokes solver as the entire process is computed on the same mesh used for the fluid computation and parallelized with Message Passing Interface for complex multi-block configurations.

\section{V.A. Modelling of Droplet Field}

An Eulerian approach is developed for modelling droplet trajectories and impingement on a solid structure. The Eulerian model solves a droplet velocity field and a volume fraction distribution in the whole computational domain. Droplets are considered spherical and not to be subject to deformation or breaking. The 
droplet phase is sufficiently diluted to neglect interactions between droplets. Temperature of the droplet phase is set to be constant and equal to free-stream temperature, heat transfer with the surrounding air is neglected. The only forces acting on droplets are drag, gravity and buoyancy.

The governing equations (which correspond to an Eulerian formulation of a Lagrangian transport of particles) for the conservation of mass and momentum of the droplets are written as follows ${ }^{94}$ :

$$
\left\{\begin{array}{l}
\frac{\partial \alpha}{\partial t}+\nabla \cdot(\alpha \mathbf{u})=0 \\
\frac{\partial \alpha \mathbf{u}}{\partial t}+\nabla \cdot(\alpha \mathbf{u} \otimes \mathbf{u})=\alpha \frac{C_{D} R e_{d}}{24 K}\left(\mathbf{u}_{a}-\mathbf{u}\right)+\alpha\left(1-\frac{\rho_{a}}{\rho}\right) \frac{1}{F r^{2}} \mathbf{g}
\end{array}\right.
$$

$\alpha$ is defined as the non-dimensionalized volume fraction of water and $\mathbf{u}$ the non-dimensionalized velocity field of droplets. $\mathbf{u}_{a}$ is the non-dimensionalized velocity of air, $\rho$ the density of water, $\rho_{a}$ the density of air, g the gravity vector. $\left.F r=U_{\infty} \sqrt{(} L g\right)$ is the Froude number, $U_{\infty}$ the freestream velocity of air, $L$ the characteristic length (typically the airfoil chord length), $K=\rho d U_{\infty} / 18 L \mu$ is an inertia parameter and $\mu$ the dynamic viscosity of air. $d$ is the median diameter of the droplets. The first term on the right-hand-side of the momentum equation accounts for the drag acting on the droplet or particle based on low-Reynolds number behaviour for spheres. The droplets Reynolds number ( $\left.R e_{d}\right)$ is defined based on the slip velocity between the air and droplet and the droplet diameter.

$$
R e_{d}=\frac{\rho d\left|\mathbf{u}_{a}-\mathbf{u}\right| U_{\infty}}{\mu}
$$

The drag coefficient of the droplets $C_{d}$ is given by :

$$
\left\{\begin{aligned}
C_{d} & =\frac{0.44}{R e_{d}}, \text { if } R e_{d}>1000 \\
C_{d} & =\frac{24}{R e_{d}}, \text { if } R e_{d}<0.1 \\
C_{d} & =\frac{24 \times\left(1+0.15 \times R e_{d}^{0.657}\right)}{R e_{d}}
\end{aligned}\right.
$$

A third order upwind scheme is used and the implicit droplet equations are solved with a BiCGSTAB algorithm. The freestream values of droplet velocity and volume fraction are imposed as boundary conditions at the far field. A switching boundary condition is applied at the wall : Neumann when the incoming droplet flux is positive and Dirichlet otherwise. Finally the collection efficiency coefficient that characterizes the configuration's ability to capture incoming water droplets on the solid surfaces is defined as :

$$
\beta=\alpha \mathbf{u} . \mathbf{n}
$$

\section{V.B. Modelling of Ice Thickness}

To calculate the properties of the ice and the liquid water film on the solid wall the mass and energy conservations are solved. The input to the equations are the wall shear stress $\tau_{\text {wall }}$ and the heat transfer coefficient $h_{c}$ obtained by the flow solver and the collection efficiency $\beta$ and the droplet velocity field $\mathbf{u}$ computed by the droplet module. The Shallow Water Icing Model icing model (SWIM) implemented is based on the Messinger model $\left({ }^{95}\right)$. The Mass and energy conservation equations are solved for each wall cell. Radiation is neglected in the energy conservation equation as it mostly accounts for a low contribution.

The system of equation writes :

$$
\left\{\begin{array}{l}
\frac{\partial \rho_{w} h}{\partial t}+\nabla \cdot\left(\rho_{w} \overline{\mathbf{u}}_{f} h\right)=\dot{m}_{i m p}-\dot{m}_{\text {evap }}-\dot{m}_{i c e} \\
\frac{\partial \rho_{w} C_{w} h T}{\partial t}+\nabla \cdot\left(\rho_{w} C_{w} \overline{\mathbf{u}}_{f} h T\right)=\dot{q}_{i m p}-\dot{q}_{e v p}-\dot{q}_{i c e}-\dot{q}_{c n v}
\end{array}\right.
$$

$h$ is the water film height, $\rho_{w}$ the water density, $C_{w}$ the specific heat of water, $T$ the surface temperature in Celsius, $\dot{m}_{\text {evp }}$ the mass rate of evaporation, $\dot{m}_{i m p}$ the impinging mass rate of water droplets and $\dot{m}_{i c e}$ the resultant mass rate of ice accretion. $\dot{q}_{i m p}$ is the heat rate of impacting droplets, $\dot{q}_{\text {evp }}$ the evaporating heat rate and $\dot{q}_{i c e}$ the heat rate of freezing water. The main contribution comes from the heat convection term $\dot{q}_{c n v}$. 
The impinging mass rate contribution writes $\dot{m}_{i m p}=L W C . V_{\infty} \cdot \beta$ and the evaporating mass rates $\dot{m}_{e v p}$ are determined by a parametric model developed by Kreith et al. ${ }^{96}$

The heat rate contributions are :

$$
\left\{\begin{array}{l}
\dot{q}_{i m p}=\dot{m}_{i m p}\left(C_{w} T_{\infty}+\frac{|\mathbf{u}|^{2}}{2}\right) \\
\dot{q}_{\text {evp }}=\dot{m}_{\text {evp }}\left(\frac{L_{e}+L_{s}}{2}\right) \\
\dot{q}_{i c e}=\dot{m}_{i c e}\left(C_{i} T-L_{f}\right) \\
\dot{q}_{c n v}=h_{c}\left(T-T_{\infty}\right) .
\end{array}\right.
$$

$C_{i}$ is the specific heat of ice, $L_{e}$ is the latent heat of evaporation, $L_{s}$ is the latent heat of sublimation and $L_{f}$ is the latent heat of fusion.

$\overline{\mathbf{u}}_{f}$ is defined as the mean velocity of the water film and writes $^{92}$ :

$$
\overline{\mathbf{u}}_{f}(\mathbf{x})=\frac{h}{2 \mu_{w}} \tau_{w a l l}(\mathbf{x})
$$

with $\tau_{\text {wall }}$ the wall shear stress, $\mathbf{x}$ the wall surfaces coordinates and $\mu_{w}$ is the viscosity of water.

To insure a physical solution and close the system, the following compatibility relations must be verified ${ }^{92}$

$$
\left\{\begin{array}{l}
h \geq 0 \\
\dot{m}_{i c e} \geq 0 \\
h T \geq 0 \\
\dot{m}_{i c e} T \leq 0
\end{array}\right.
$$

The heat transfer coefficient is a key parameter in icing simulations. In NSMB, this coefficient is evaluated from the heat fluxes $q_{\text {wall }}$ by imposing a constant wall temperature $T_{\text {wall }}$. The analytical definition of the heat transfer coefficient writes :

$$
h_{c}=\frac{q_{\text {wall }}}{T_{\infty}-T_{\text {wall }}} .
$$

The heat transfer coefficient is highly dependent to the chosen roughness parameter. As a first approximation, the constant equivalent sand grain roughness parameter used in this paper is determined by the correlation from Shin and Bond. ${ }^{97}$

The convective terms of the Eq. 6 are discretized via finite volume method with a first order Roe scheme. The system of two equations is solved explicitly in time via a Runge-Kutta scheme and is parallelized with MPI.

\section{V.C. Results}

Rime icing is simulated on a NACA23012 airfoil and compared with experimental result from Broeren et al. ${ }^{98}$ The O-grid mesh is generated by NS-GRID software presently developed by Ecole Polytechnique de Montreal. NS-GRID is an elliptic smoothing mesh generation code that control orthogonality, skewness and spacings via control functions. ${ }^{91}$ The NACA23012 mesh has a chord of $c=1.828 m$ and contains $512 \times 256$ cells divided into two blocks with a farfield extended to 50 chords.

The parameters of the simulations are an angle of attack of $2.0^{\circ}$, the Mach number is $M a=0.24$, the Reynolds number is $R e=12.60 \times 10^{6}$, the freestream temperature is $T=247.85 \mathrm{~K}$, the median droplet diameter is $d=30 \mu \mathrm{m}$, the liquid water content is $L W C_{\infty}=0.55 \mathrm{~g} / \mathrm{m}^{3}$ and the total icing time is $600 \mathrm{~s}$. Visualization of Liquid Water Content distribution is shown in Fig. 19. Ice shape obtained after a single icing step are compared with experimental results in Fig. 20. The simulated ice shape is in excellent agreement with experimental data. One should not that taking into account a droplet size distribution to simulate the water droplet spray should provide even more precise results on the upper impingement limit where a slight 
underestimation of the ice thickness is observed.

Glaze icing is simulated on a two-dimensional NACA0012 airfoil and compared with NASA LEWICE2D ${ }^{99}$ and the experimental data available. ${ }^{100}$ The NACA0012 O-grid mesh was generated by NS-GRID and has a chord of $c=0.5334 \mathrm{~m}$. The mesh contains $512 \times 256$ cells divided in two blocks. The constant sand grain roughness used in the computation is set to $k_{s}=0.55 \mathrm{~mm}$. Calculation is performed on two cores. Impingement efficiency distribution curve along the airfoil surface is shown on Fig. 21. Compared to LEWICE, present results showcase a slight higher value of the lower impingement limits. This observation is in accordance with results of several studies of the Eulerian droplet model. ${ }^{94,101}$

The calculated glaze-type ice shape is generated with three icing steps and compared with available data in Fig. 22. This test was repeated on a NACA00012 extruded mesh. Identical results were obtained along wing span partially validating the three-dimensional implementation.

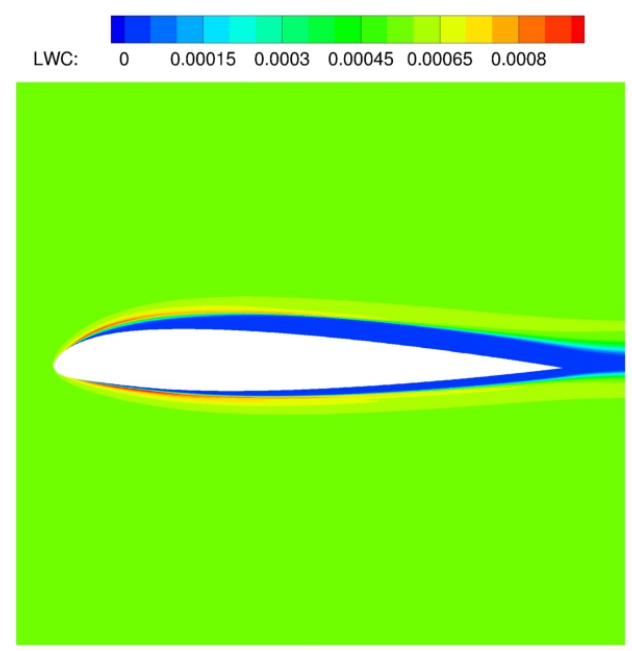

Figure 19: NACA23012 - Visualization of Liquid Water Content. ${ }^{5}$

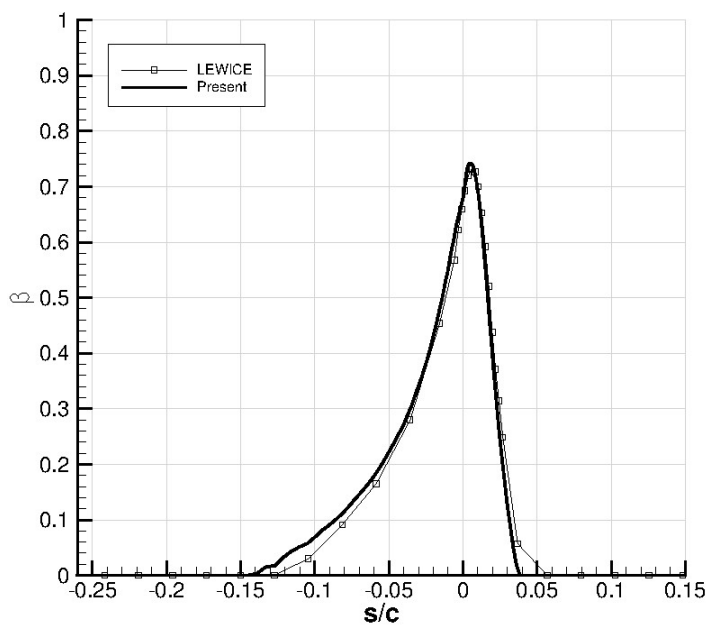

Figure 21: NACA00012 - Comparison of collection efficiency with LEWICE on clean geometry. ${ }^{5}$

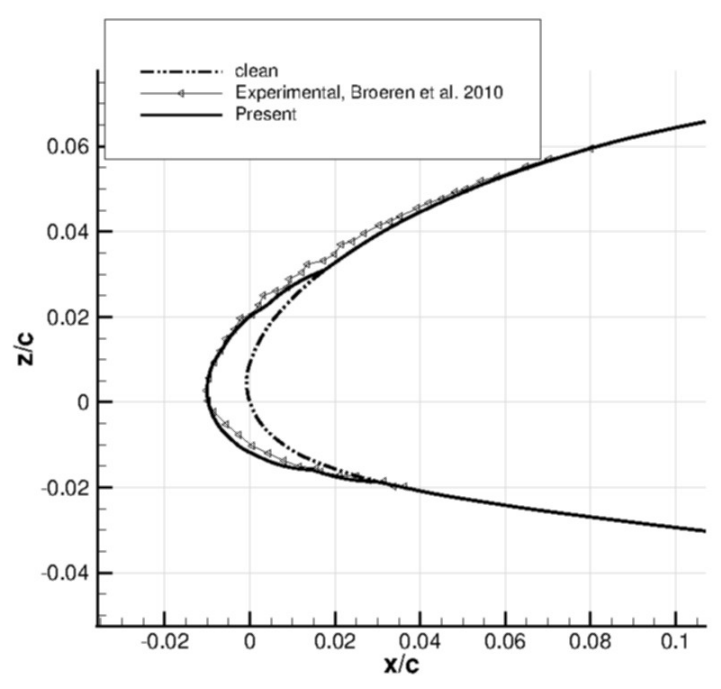

Figure 20: NACA23012 - Comparison of ice shape with experimental data from, ${ }^{985}$

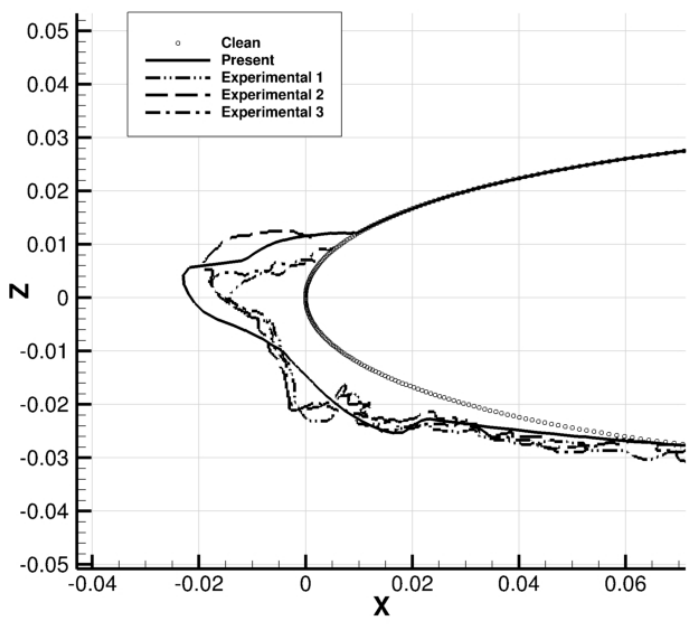

Figure 22: NACA00012 - Comparison of ice shape with experimental data from. ${ }^{100}$

The last test case is a three-dimensional simulation performed on an ONERA-M6 swept wing. The 4 blocks mesh contains 884736 cells. The mean aerodynamic chord is $c=0.53 \mathrm{~m}$, the semi-span is $b=1 \mathrm{~m}$ 
and the computational domain extends to $20 \times c$. The flow is simulated at an angle of attack of $6^{\circ}$, a Mach number of $M=0.15$, a Reynolds number of $R e=2.17 \times 10^{6}$, a static temperature of $T=265 K$ and with a constant equivalent sand grain roughness number of $k_{s}=0.55 \mathrm{~mm}$. The parameters of the water spray are a mono-dispersed droplet diameter of $d=20 \mu \mathrm{m}$ and a liquid water content of $L W C=1.0 \mathrm{~g} / \mathrm{m}^{3}$. Icing is simulated at a total time of $t=500 \mathrm{~s}$.

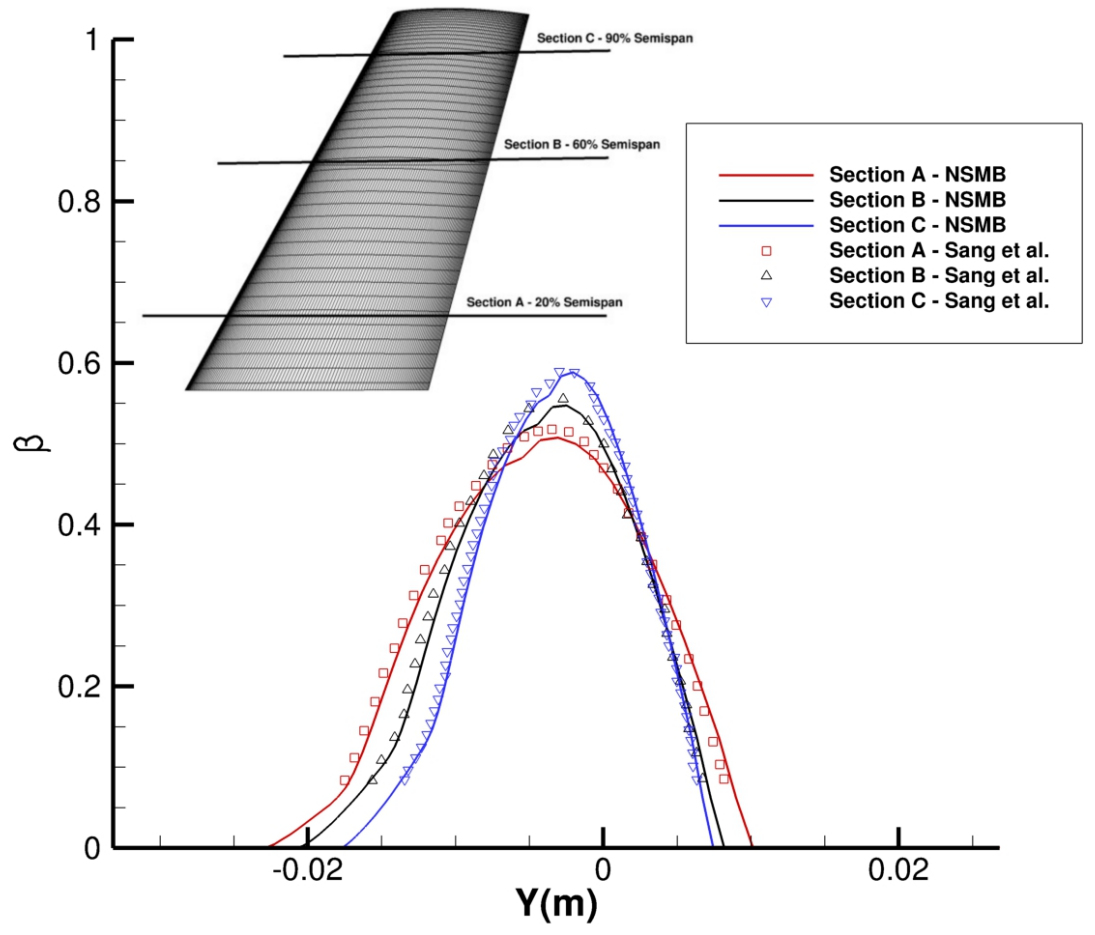

Figure 23: Collection efficiency on an ONERA M6 swept wing ${ }^{5}$

Impingement curves are plotted on three sections A, B \& C respectively at 20\%, $60 \%$ and $90 \%$ of span length and compared with Lagrangian results from Sang et al. ${ }^{102}$ in Fig. 23. The present results show a good agreement. The three-dimensional ice shape at exposure time $t=500 \mathrm{~s}$ is represented in Fig. 24 . According to our knowledge there is unfortunately no experimental or numerical data regarding ice shapes to compare with on this configuration.

\section{2nd High Lift Prediction Workshop}

NSMB was used to perform studies in the 2nd AIAA High Lift Prediction Workshop (HiLiftPW2) to better understand the influence of the numerical schemes on numerical predictions. ${ }^{103}$ The model is the DLR- F11 model (Figure 25), a semi-span three element high lift wing representative for a commercial wide-body twin-jet. 104

While all participants ran the mandatory test cases in RANS mode, NSMB was one of few that ran URANS simulations. This allowed characterizing the flow in steady or unsteady states. In addition, emphasis was placed on the study of effects of initial conditions on final solution.

The grid is the original 1-to-1 structured grid provided by the HiLiftPW-2 committee. Three grid sizes are proposed: coarse, medium and fine with respectively 9.5, 32 and 101 million cells. Each grid level is obtained by a uniform growth of around 1.5 time in each coordinate direction. The non-dimensional normal 


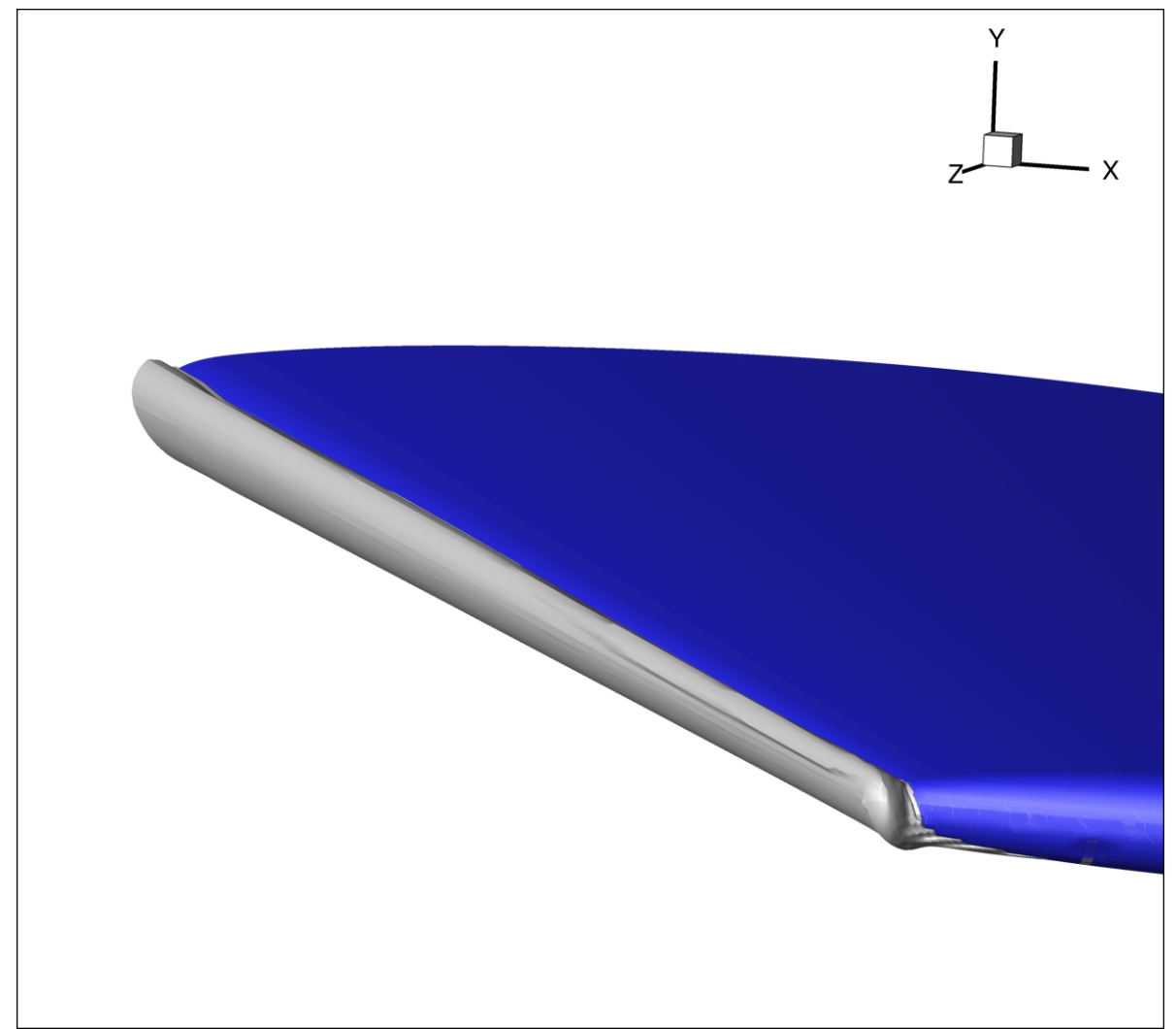

Figure 24: Icing on a ONERA M6 swept wing

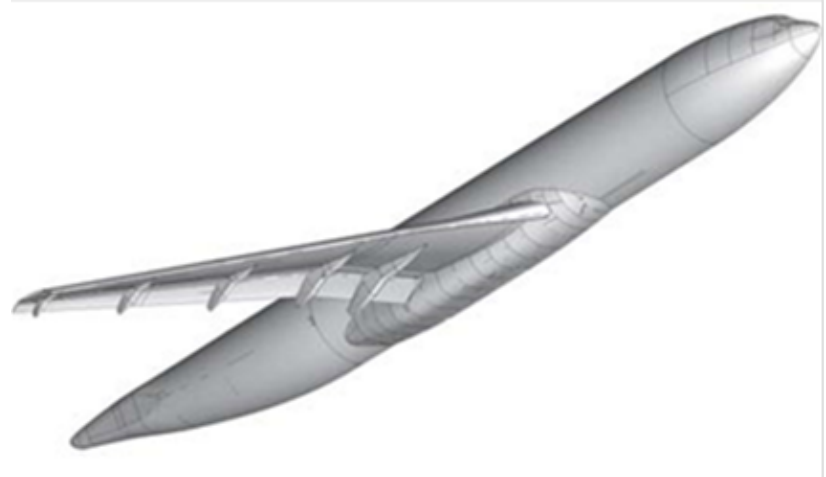

Figure 25: DLR-F11 geometry. 
wall spacing of the first cell is $1.8810^{6}, 1.2910^{6}$ and $0.8310^{6}$ respectively for the coarse, medium and fine grid, and the farfield is located at around 100 reference chords.

NSMB settings were : second order central scheme for diusive terms, fourth order central scheme with artficial dissipation of Jameson for convective terms. For unsteady calculations, a dual time stepping scheme with backward second order interpolation is used. The system is solved with an implicit LU-SGS (Lower-Upper Symmetric Gauss Seidel) scheme with 100 inner iterations with 2 level multigrid. The nondimensionalized timestep was 0.06 . Three variant of the fully turbulent Spalart-Allmaras turbulent model are tested : the original Spalart-Allmaras and the Edwards and SALSA modifications.

Here, only results concerning the effect of initial conditions are discussed. The dataset is as listed in table 1, all referring to the medium mesh and using the steady solver with the SA-Edwards turbulence model.

\begin{tabular}{|l|l|}
\hline Angle of attacks & Initial conditions \\
\hline $20,21,22,24$ & freestream \\
\hline $18.5,19,19.5,20,20.5,21,21.5,22,22.4,23,24$ & from alpha=16 deg \\
\hline $17,18,19,20,21$ & from alpha=alpha $-1 \mathrm{deg}$ \\
\hline & \\
\hline
\end{tabular}

Table 1: Dataset for initial condition study.

The trend of the lift coefficient is plotted in figure 26 for the three initial conditions. The difference between the initial conditions occurs only after $\alpha=21^{\circ}$. After this angle-of-attack, the lift is overpredicted with the results initialized from freestream, whereas the opposite occurs with the initial condition set at 16 degrees. The global trend of the lift differs from that observed in the experiment. The stall curve presents a drought around 20 degrees before the maximum lift for all initial conditions. An examination of the wing surface pressure distributions shows that this drought is related to the fuselage/wing junction. A linear eddy viscosity model using the Boussinesq relation can be replaced by a nonlinear stress term like the Quadratic Constitutive Relation (QCR), a scheme described by Spalart and applied to the HiLiftPW-2 by Pulliam \& Sclafani ${ }^{105}$.

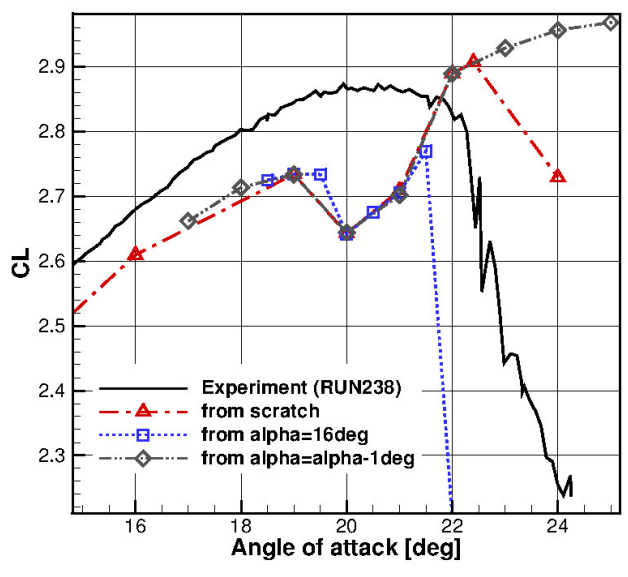

a)

Figure 26: Lift coefficient (a) and its amplitude (b) versus the angle of attack for several initial conditions.

\section{Recent developments for hypersonic flow simulations}

NSMB code was from the start of its development designed for use for hypersonic applications (in particular re-entry space vehicles). NSMB includes different levels of chemistry modeling for Air, CO2 and N2. Available models for air consider 5, 7, 11 or 13 chemical species, and a large number of non-equilibrium chemistry models are available, among them the models of Park 1989, ${ }^{106}$ Park $1993,{ }^{107}$ Park 2001, ${ }^{108}$ Gar- 
diner ${ }^{109}$ and Dunn and Kang ${ }^{110}$ are available. For all these models the equilibrium constants for the chemical reactions are computed using the polynomials provided in the references.

Chemical equilibrium can be computed using polynomials (TGAS ${ }^{111} / \mathrm{VGAS}^{112}$ ), or by calculating the chemical composition for equilibrium.

Thermochemical non-equilibrium has been implemented using the Landau-Teller equation for the translationalvibrational energy exchange with the relaxation time calculated using the semi empirical Millikan and White formula. All these models are available for inviscid and viscous (both laminar and turbulent) flows.

Transport coefficients are computed using the Blottner model ${ }^{113}$ or the Gupta-Yos model. ${ }^{114}$

Different methods of catalytic wall boundary conditions have been implemented, including super catalytic walls and partially catalytic walls.

NSMB includes an option to adapt the far field boundary to the bow shock. This option removes the so-called carbuncle phenomenon introduced by the numerical scheme due to the non-alignment of the grid with the bow shock. The bow shock adaption procedure requires a C-type of grid, and the shock should be located in the blocks with a far-field boundary condition. During the solution process the position of the shock on a grid line is determined using the Mach number as variable (other variables were used but were less accurate or less robust). Once the shock position is known the far field boundary is moved along the original grid line to a position near the shock location. Then grid lines are clustered around the shock position, followed by a redistribution of the grid points on the remainder of the original grid line. In general the grid needs to be adapted two to three times to obtain a stable shock position.

For capsule type geometries the bow shock grid adaption improves considerably the solution on the heat shield, and in particular the heat flux. Figure 27 shows the Mach number contours in the symmetry plane for the ARV capsule, and one clearly observes the improvement due to the grid adaptation.

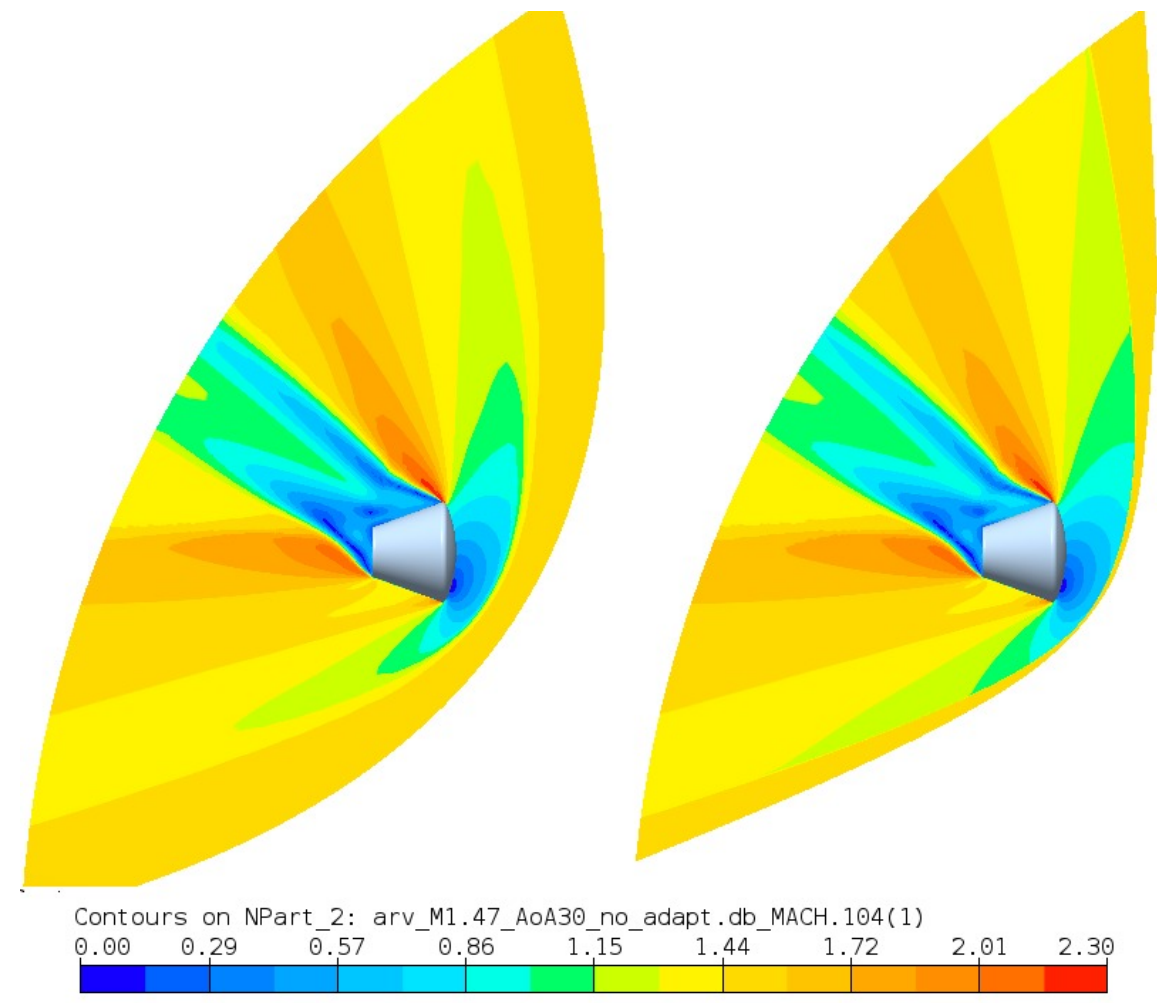

Figure 27: Mach number contours symmetry plane ARV capsule at Mach $=1.47$, left unadapted grid, right adapted grid.

Figure 28 shows the heat flux contours on the heat shield for a chemical non-equilibrium calculation at Mach $=25.1$ for the original and adapted grid. One clearly observes the improvements in the heat flux due to the adaption of the grid to the bow shock.

Roughness and blowing modeling was implemented in NSMB during the European Union funded project 

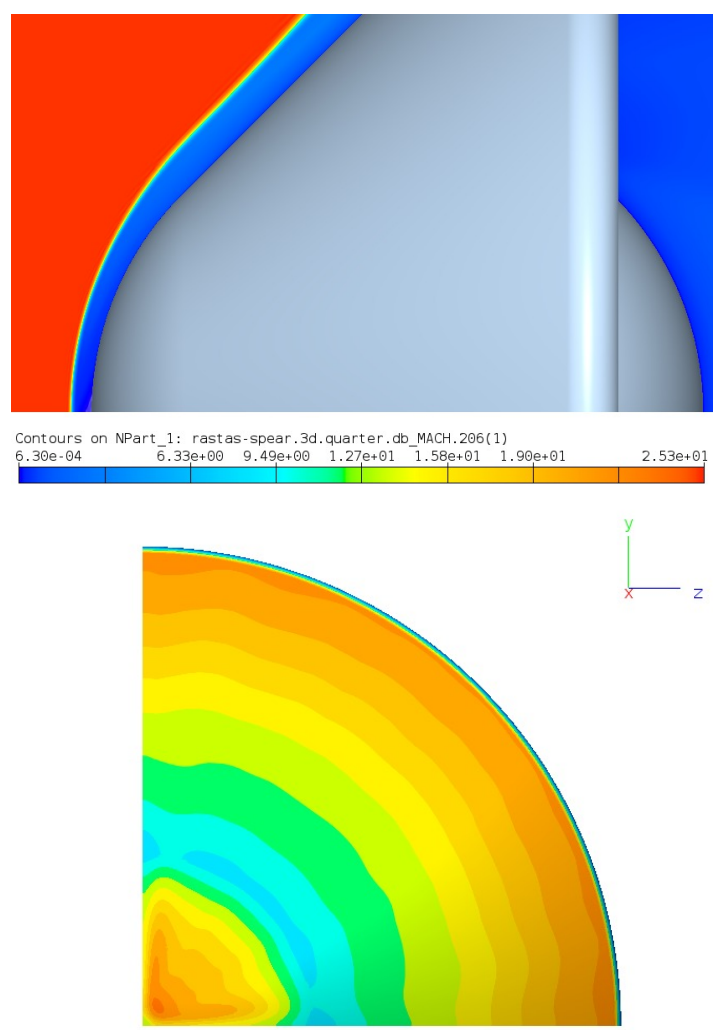

Contours on NPart 1: rastas-spear.3d.quarter. db $\mathrm{CH} .206 \quad 1(1)$ $\odot .00 \mathrm{e}+00 \quad 1.25 \mathrm{e}+06 \quad 12.50 \mathrm{e}+06 \quad 3.75 \mathrm{e}+06 \quad 5.00 \mathrm{e}+06$
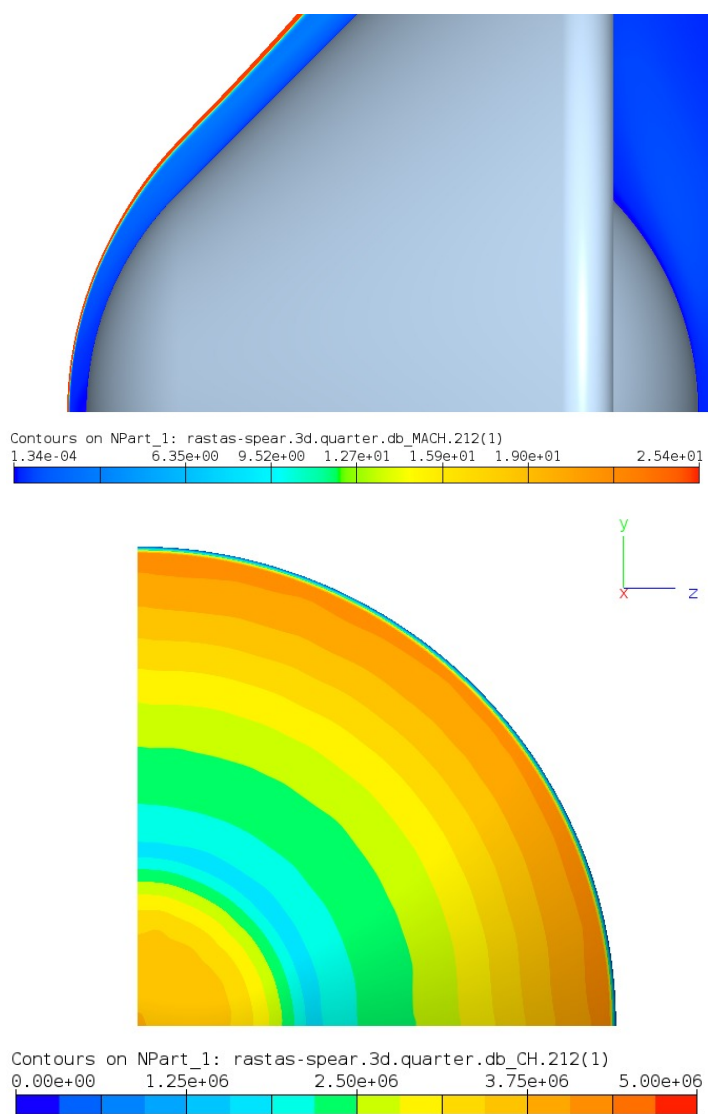

Figure 28: Mach numbers in the symmetry plane (top row) and heat flux on the heat shield (bottom row), unadapted grid (left) and twice adapted grid (right). Earth Re-entry Vehicle at Mach $=25.1, h=48 \mathrm{~km}$, chemical non-equilibrium simulation using a 13 species air model. 
RASTAS-SPEAR (Radiation-Shapes-Thermal Protection Investigations for High Speed Earth Re-entry),. ${ }^{4}$ For roughness modeling the so-called equivalent sand-grain approach is used, which consists of two steps. In the first step the rough surface is reduced to an equivalent reference surface, in the second step the turbulence model is modified to reproduce the effect of rough surfaces on the skin friction and heat transfer. Reviews of surface roughness modeling can be found in. ${ }^{115-117}$ Both the Spalart-Allmaras turbulence model ${ }^{61}$ and the $k-\omega$ family of turbulence models ${ }^{66,67}$ were modified to account for rough walls.

Calculations were made for the Earth Re-entry Vehicle shown in Fig. 28 using the Spalart-Allmaras turbulence model for a smooth wall for a rough wall using 2 roughness heights $(250$ and $500 \mu \mathrm{m})$. Figure 29 shows the computed heat flux along the wall in the symmetry plane. No blowing was used for these calculations. The computed results are compared with empirical correlations for a laminar flow ${ }^{118}$ and a turbulent flow. ${ }^{119}$ Roughness effect are taken into account in the empirical correlations using the formulation of Powars. ${ }^{120}$

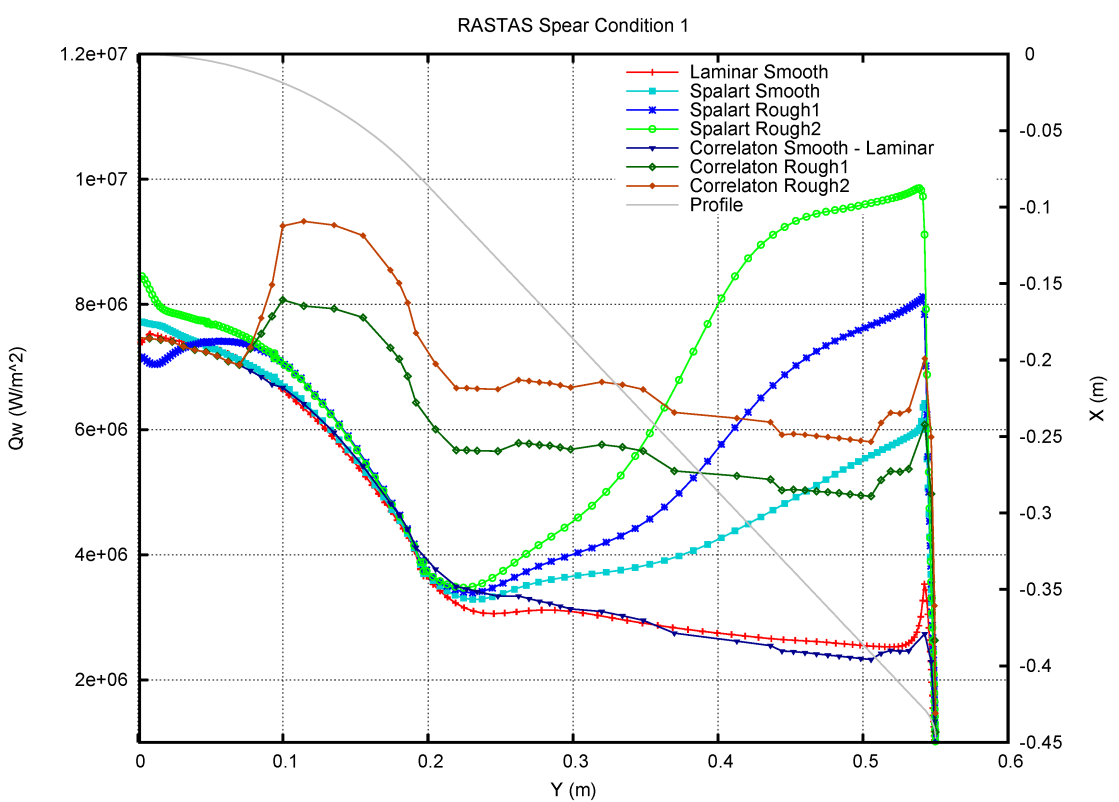

Figure 29: Influence of surface roughness on the heat flux, Earth Re-entry Vehicle at Mach $=25.1, h=$ $48 \mathrm{~km}$, chemical non-equilibrium calculation. 
As can be seen in Fig. 29, for a laminar flow the computed heat flux is in excellent agreement with the heat flux obtained from empirical correlations. For a turbulent flow using the Spalart Allmaras turbulence model the computed heat flux follows the laminar result until $y=0.23 \mathrm{~m}$, indicating that the flow at the nose is laminar and that transition to turbulence takes place further downstream. Once transition takes place the heat flux increases rapidly. This increase in heat flux is faster and higher when using roughness modeling. The turbulent correlations also assume a laminar flow at the nose (until $y=0.08 \mathrm{~m}$ ), followed by a turbulent flow further downstream.

Table 2 summarizes the increase in heat flux compared to a smooth wall at 4 different points.

\begin{tabular}{|l|l|l|l|l|}
\hline & Mid Sphere & $\begin{array}{l}\text { Sphere-Cone } \\
\text { Junction } \\
Y=0.2 \mathrm{~m}\end{array}$ & Mid-cone & Tore \\
& $Y=0.1 \mathrm{~m}$ & $Y .37 \mathrm{~m}$ & \\
\hline Spalart Rough 1 & $5 \%$ & $2 \%$ & $25 \%$ & $27 \%$ \\
\hline Spalart Rough 2 & $5 \%$ & $2 \%$ & $71 \%$ & $54 \%$ \\
\hline Correlation Rough 1 & $21 \%$ & $21 \%$ & $20 \%$ & $22 \%$ \\
\hline Correlation Rough 2 & $39 \%$ & $42 \%$ & $41 \%$ & $43 \%$ \\
\hline
\end{tabular}

Table 2: Influence roughness on computed heat fluxes.

In a recent project funded by the European Space Agency on Ablation modeling NSMB was coupled to the Mutation++ library developed at the Von Karman Institute. ${ }^{121}$ This library is written in C++ and designed to provide efficient algorithms for the computation of various properties important in the fields of hypersonics and combustion Computational Fluid Dynamics (CFD) including:

- Thermodynamic Properties using

- The statistical mechanics approach using partition functions for the different energy modes of a species. Rigid-Rotor and Harmonic Oscillator models have been implemented

- Multi-temperature lookup tables for high fidelity models based on quantum mechanics

- High fidelity curve fits, and in particular the NASA 7 and 9 coefficient polynomial data bases

- Multicomponent Transport Properties

- Use of collision integrals for each collision pair of species in the mixture

- Wilke, Gupta-Yos, Armaly-Sutton and Blottner mixture rules

- State of the art methods to solve the system, LDLT for direct solution and Conjugate-Gradient for iterative solution

- Finite Rate Chemistry in Thermal Non-equilibrium

- A highly robust Multiphase Equilibrium solver (not used by NSMB).

In the next phase of the project an engineering model will be developed to account for both carbonbased TPS ablation and catalysis. These models will be updated and refined using experiments carried out at the Von Karman Institute in Belgium. Once this process is complete these models will be included in Mutation++, and can then be used by the NSMB CFD code to perform CFD simulations of re-entry vehicles using ablative TPS materials.

\section{Conclusion}

Since the last AIAA paper describing the NSMB solver ${ }^{1}$, the NSMB developpers consortium has been reduced but the development of the this code is still on-going and many major improvements have been performed in the last few years : an fully automatic chimera method for moving bodies, an icing model, many turbulence models for unsteady flows and new models for hypersonic flows. All these developments have been successfully applied to various flow configuration allowing the NSMB solver to be one of the most powerfull research CFD solver in Europe. 


\section{References}

${ }^{1}$ Vos J.B., Rizzi A.W., Corjon A., Chaput E., Soinne E. Recent Advances in Aerodynamics inside the NSMB (Navier-Stokes Multiblock) Consortium. AIAA paper 98-0225, 1998.

${ }^{2}$ Guillaume M., Gehri A., Stephani P., Vos J., Manadanis G., Fluid Structure Interaction Simulation on the F/A-18 Vertical Tail, AIAA-2010-4613, Chicago, 2010.

${ }^{3}$ Vos J.B., Sanchi S., Gehri A., Drag Prediction Workshop 4 Results Using Different Grids Including Near-Field/Far-Field Drag Analysis, Journal of Aircraft, Vol. 50, No. 5, 2013.

${ }^{4}$ J.B. Vos, A. Bourgoing, J. Soler, B. Rey. Earth re-entry capsule CFD simulations taking into account surface roughness and mass injection at the wall, Paper accepted for publication in Int. Journal Aerodynamics.

${ }^{5}$ D. Pena, E. Laurendeau, Y. Hoarau.Development of a Three-Dimensional Icing Simulation Code in the NSMB Flow Solver, Paper accepted for publication in Int. J. of Engineering Systems Modelling and Simulation

${ }^{6}$ F. Grossi, M. Braza, Y. Hoarau. Prediction of Transonic Buffet by Delayed Detached-Eddy Simulation, AIAA Journal, Vol. 52, No. 10, pp. 2300-2312, 2014

${ }^{7}$ V. Shinde, T. Marcel, Y. Hoarau, T. Deloze, G. Harran, F. Baj, J. Cardolaccia, J.P. Magnaud, E. Longatte, M. Braza. Numerical simulation of the fluidstructure interaction in a tube array under cross flow at moderate and high Reynolds number, J. of Fluids and Structures, Vol 47, pp. 99-113, 2014

${ }^{8}$ T. Deloze, Y. Hoarau, J.Dusek. Transition scenario of a sphere freely falling in a vertical tube, J. Fluid Mechanics, Vol 711, pp. 40-60, 2012

${ }^{9}$ T. Deloze, Y. Hoarau, J.Dusek. Chimera method applied to the simulation of a freely falling cylinder in a channel, European Journal of Computational Mechanics, Vol 19/5-7, pp.575-590, 2010

${ }^{10}$ J. A. Benek, J. L. Steger, and F.C. Dougherty. A flexible grid embedding technique with application to the euler equations. AIA A Paper, 83-1944, 1983.

${ }^{11}$ W.D. Henshaw and D.W. Schwendeman. An adaptive numerical scheme for high-speed reactive flow on overlapping grids. Journal of Computational Physics, 191(2):420-447, 2003.

${ }^{12}$ W.D. Henshaw and D.W. Schwendeman. Moving overlapping grids with adaptive mesh refinement for high-speed reactive and non-reactive flow. Journal of Computational Physics, 216(2):744-779, 2006.

${ }^{13}$ AK Kapila, DW Schwendeman, JB Bdzil, and WD Henshaw. A study of detonation diffraction in the ignition-and-growth model. Combustion Theory and Modelling, 11(5):781-822, 2007.

${ }^{14}$ PG Buning, IT Chiu, S. Obayashi, YM Rizk, and JL Steger. Numerical simulation of the integrated space shuttle vehicle in ascent. In AIAA Atmospheric Flight Mechanics Conference, Minneapolis, MN, pages 265-283, 1988.

${ }^{15} \mathrm{M}$. Hinatsu and JH Ferziger. Numerical computation of unsteady incompressible flow in complex geometry using a composite multigrid technique. International Journal for Numerical Methods in Fluids, 13(8):971-997, 1991.

${ }^{16}$ R.L. Meakin. Moving body overset grid methods for complete aircraft tiltrotor simulations. In AIAA Computational Fluid Dynamics Conference, 11 th, Orlando, FL, Technical Papers. Pt. 2, volume 6(9), 1993.

${ }^{17}$ D.G. Pearce, S.A. Stanley, F.W. Martin Jr, R.J. Gomez, G.J. Le Beau, P.G. Buning, W.M. Chan, I.T. Chiu, A. Wulf, and V. Akdag. Development of a large scale Chimera grid system for the space shuttle launch vehicle. In AIAA, 31st Aerospace Sciences Meeting, Reno, NV, volume 0533, 1993.

${ }^{18} \mathrm{R}$. Maple and D. Belk. A new approach to domain decomposition, the beggar code. Numerical Grid Generation in Computational Fluid Dynamics and Related Fields, Pineridge Press Limited, pages 305-314, 1994.

${ }^{19}$ D. Jespersen, TH Pulliam, and P. Buning. Recent enhancements to OVERFLOW. AIAA paper, 97-0644, 1997.

${ }^{20} \mathrm{R}$. L. Meakin. Chapter 11 : Composite overset structured grids, in : Handbook of grid generation, J.F. Thompson, B.K. Soni and N.P. Weatherill. CRC, 1999.

${ }^{21}$ R.L. Meakin and N.E. Suhs. Unsteady aerodynamic simulation of multiple bodies in relative motion. In AIAA Computational Fluid Dynamics Conference, 9 th, Buffalo, NY, volume AIAA-89-1996, pages 643-657, 1989.

${ }^{22}$ W.D. Henshaw and D.W. Schwendeman. Parallel computation of three-dimensional flows using overlapping grids with adaptive mesh refinement. Journal of Computational Physics, 227:7469-7502, 2008.

${ }^{23}$ B. Landmann and M. Montagnac. A highly automated parallel Chimera method for overset grids based on the implicit hole cutting technique. International Journal for Numerical Methods in Fluids, 2010.

${ }^{24}$ R.L. Meakin. A new method for establishing intergrid communication among systems of overset grids. AIAA Paper, 91-1586, 1991.

${ }^{25}$ H. Samet. The design and analysis of spatial data structures. Addison-Wesley, 1990.

${ }^{26}$ Timo L. Siikonen, Patrick P. Rautaheimo, and Esa J. Salminen. Numerical techniques for complex aeronautical flows. European Congress on Computational Methods in Applied Sciences and Engineering ECCOMAS 2000, 2000.

${ }^{27}$ Wei Liao, Jinsheng Cai, and Her Mann Tsai. A multigrid overset grid flow solver with implicit hole cutting method. Computer Methods in Applied Mechanics and Engineering, 196:1701 - 1715, 2007.

${ }^{28}$ Jubaraj Sahu and Joseph L. Steger. Numerical simulation of three-dimensional transonic flows. International Journal for Numerical Methods in Fluids, 10(8):855-873, 1990.

${ }^{29}$ G. Desquesnes, M. Terracol, E. Manoha, and P. Sagaut. On the use of a high order overlapping grid method for coupling in CFD/CAA. Journal of Computational Physics, 220:355-382, 2006.

${ }^{30}$ J.W. Delfs. An overlapped grid technique for high resolution CAA schemes for complex geometries. AIAA Paper, Seventh AIAA/CEAS Aerocoustics Conference, Reno, May 2001, 2001-2199, 2001.

${ }^{31}$ S.E. Sherer and J.N. Scott. High-order compact finite-difference methods on general overset grids. Journal of Computational Physics, 210:459-496, 2005.

${ }^{32}$ T.K. Sengupta, VK Suman, and N. Singh. Solving Navier-Stokes equation for flow past cylinders using single-block structured and overset grids. Journal of Computational Physics, 229(1):178-199, 2010. 
${ }^{33}$ G. Chesshire and WD Henshaw. Composite overlapping meshes for the solution of partial differential equations. Journal of Computational Physics, 90:1-64, 1990.

${ }^{34}$ HS Tang and T. Zhou. On nonconservative algorithms for grid interfaces. SIAM Journal on Numerical Analysis, 37:173-193, 1999 .

${ }^{35}$ CJ Freitas and SR Runnels. Simulation of fluid-structure interaction using patched-overset grids. Journal of fluids and Structures, 13:191-207, 1999.

${ }^{36} \mathrm{Z}$ J Wang. A fully conservative interface algorithm for overlapped grids. Journal of Computational Physics, 122:96-106, 1995.

${ }^{37}$ Man Mohan Rai. A relaxation approach to patched-grid calculations with the euler equations. Journal of Computational Physics, 66:99 - 131, 1986 .

${ }^{38}$ JA Wright and W. Shyy. A pressure-based composite grid method for the Navier-Stokes equations. Journal of computational physics, 107:225-238, 1993.

${ }^{39}$ HS Tang, S. Casey Jones, and F. Sotiropoulos. An overset-grid method for 3D unsteady incompressible flows. Journal of Computational Physics, 191:567-600, 2003.

${ }^{40}$ W.D. Henshaw. A fourth-order accurate method for the incompressible Navier-Stokes equations on overlapping grids. Journal of Computational Physics, 113:13-25, 1994.

${ }^{41}$ Y. Zang and RL Street. A composite multigrid method for calculating unsteady incompressible flows in geometrically complex domains. International Journal for Numerical Methods in Fluids, 20:341-362, 1995.

${ }^{42} \mathrm{~B}$. Hubbard and H. C. Chen. A chimera scheme for incompressible viscous flows with application to submarine hydrodynamics. AIAA Paper, 25th AIAA Fluid Dynamics Conference, 2210, 1990.

${ }^{43}$ Jean-Jacques Chattot and Y. Wang. Improved treatment of intersecting bodies with the chimera method and validation with a simple and fast flow solver. Computers \& fluids, 27:721-740, 1998.

${ }^{44}$ W. Liao, J. Cai, and H.M. Tsai. A multigrid overset grid flow solver with implicit hole cutting method. Computer Methods in Applied Mechanics and Engineering, 196:1701-1715, 2007.

${ }^{45}$ T.M. Burton and J.K. Eaton. Analysis of a fractional-step method on overset grids. Journal of Computational Physics, 177:336-364, 2002.

${ }^{46} \mathrm{CH}$ Tai, Y. Zhao, and KM Liew. Parallel computation of unsteady incompressible viscous flows around moving rigid bodies using an immersed object method with overlapping grids. Journal of Computational Physics, 207:151-172, 2005.

${ }^{47}$ A. Pigeon. Développement d'une méthode d'accélération par grilles virtuelles récursives pour l'assemblage de maillages chimères. Mémoire de maitrise, École Polytechnique de Montréal, 2015

${ }^{48}$ A. T. Levesque, A. Pigeon, T. Deloze, E. Laurendeau. An Overset Grid 2D/Infinite Swept Wing URANS Solver Using Recursive Cartesian Bucket Method. AIAA Scitech, Jan. 2015.

${ }^{49} \mathrm{~S}$. Taneda. Experimental investigation of the wake behind a sphere at low Reynolds numbers. J. Phys. Soc. Japan, 11(10):1104-1108, 1956.

${ }^{50}$ RH Magarvey and R.L. Bishop. Transition ranges for three-dimensional wakes. Canadian Journal of Physics, 39(10):14181422,1961

${ }^{51}$ M. Provansal and D. Ormieres. Transition to turbulence in the wakes of axisymmetrical objects. In Symposium on Turbulent Shear Flows, 11 th, Grenoble, France, pages 17-7, 1997.

${ }^{52} \mathrm{G}$. Bouchet, M. Mebarek, and J. Dušek. Hydrodynamics forces acting on a rigid fixed sphere in early transitional regimes. European Journal of Mechanics B/Fluids, 25:321-336, 2006.

${ }^{53}$ TA Johnson and VC Patel. Flow past a sphere up to a Reynolds number of 300. Journal of Fluid Mechanics, 378:19-70, 1999.

${ }^{54}$ B. Ghidersa and Jzn Dušek. Breaking of axisymmetry and onset of unsteadiness in the wake of a sphere. Journal of Fluid Mechanics, 423:33-69, 2000.

${ }^{55}$ A.G. Tomboulides and S.A. Orszag. Numerical investigation of transitional and weak turbulent flow past a sphere. Journal of Fluid Mechanics, 416:45-73, 2000.

${ }^{56}$ I. Nakamura. Steady wake behind a sphere. Physics of Fluids, 19:5, 1976.

${ }^{57} \mathrm{FW}$ Roos and WW Willmarth. Some experimental results on sphere and disk drag. AIAA Journal, 9:285-291, 1971.

${ }^{58}$ R. Mittal. Planar symmetry in the unsteady wake of a sphere. AIAA journal, 37(3):388-390, 1999.

${ }^{59} \mathrm{P}$. Bagchi and S. Balachandar. Steady planar straining flow past a rigid sphere at moderate Reynolds number. Journal of Fluid Mechanics, 466:365-407, 2002.

${ }^{60}$ Lanying Zeng, S. Balachandar, and Paul Fischer. Wall-induced forces on a rigid sphere at finite Reynolds number. J. Fluid Mech., 536:1-25, 2005.

${ }^{61}$ P.R. Spalart S.R. and Allmaras. A One-Equation Turbulence Model for Aerodynamic Flows, AIAA Paper 92-0439, Jan. 1992.

62 J.R. Edwards and S. Chandra. Comparison of eddy viscosity-transport turbulence models for three-dimensional, shockseparated flowfields. AIAA Journal 34 (9), 756763, 1996.

${ }^{63}$ M. Shur, M. Strelets, L. Zaikov, A., Gulyaev, V. KozIov, A. Secundov. Comparative numerical testing of one- and twoequation turbulence models for flows with separation and reattachement. In: 33rd Aerospace Sciences Meeting and Exhibit, Reno, NV, USA, AIAA 95-0863, 912 January, 1995.

${ }^{64}$ P.R. Spalart. Trends in turbulence treatments. In: Fluids 2000 Conference and Exhibit, Denver, CO, USA, AIAA Paper 20002306, 1922 June, 2000.

${ }^{65}$ K.Y. Chien. Predictions of channel and boundary-layer flows with a low-Reynolds number turbulence model, AIAA Journal, vol. 20, pp.33-38, 1982.

${ }^{66}$ Wilcox D.C., Turbulence Modeling for CFD, DCW Industries, Inc. La Cañada, California, 1993

${ }^{67}$ Menter F.R., Zonal Two-equation k-w Turbulence Models for Aerodynamics Flows, AIAA Paper, 93-2906, 1993 
${ }^{68}$ K. Abe, Y.-J. Jang and M.A. Leschziner. An investigation of wall-anisotropy expressions and length-scale equations for non-linear eddy-viscosity models, Int. J. of Heat and Fluid Flow, vol. 24, pp. 181-198, 2003.

${ }^{69}$ B.E. Launder, G.J. Reece and W. Rodi. Progress in the development of a Reynolds-stress turbulence closure, J. of Fluid Mech, vol. 68, pp. 537-566, 1975.

${ }^{70}$ N. Shima. Low-Reynoldsnumberr Second-Moment Closure Without Wall-Reflection Redistribution Terms, Int. J. of Heat and Fluid Flow, vol. 19, pp. 549-225, 1998.

${ }^{71}$ C. G. Speziale, T. B. Gatski and S. Sarkar. Modeling the Pressure-Strain Correlation of Turbulence: an Invariant Dynamical Systems Approach, J. of Fluid Mechanics,vol. 227, pp. 245-272, 1991.

${ }^{72}$ F.R. Menter and Y. Egorov.Scale-Adaptive Simulation Method for Unsteady Flow Predictions. Part 1: Theory and Model Description, Flow, Turbulence and Combustion, vol. 85, vol. 1, pp. 113-138, 2010.

${ }^{73}$ M. Braza, R. Perrin, Y. Hoarau. Turbulence properties in the cylinder wake at high Reynolds number, J. of Fluids and Structures, vol. 22 (6-7), pp. 757-771, 2006.

${ }^{74}$ R. Bourguet, M. Braza, G. Harran, R. El Akoury. Anisotropic organised eddy simulation for the prediction of nonequilibrium turbulent flows around bodies, J. of Fluids and Structures, vol. 24 (8), pp. 1240-1251, 2008.

${ }^{75}$ W. Haase, M. Braza, A. Revell. DESider A European Effort on Hybrid RANS-LES Modelling: Results of the EuropeanUnion Funded Project, 2004-2007, Notes on Numerical Fluid Mechanics and Multidisciplinary Design, vol. 103, Springer, 2009.

${ }^{76}$ P.R. Spalart, W.-H Jou, M. Stretlets, S.R. Allmaras. Comments on the Feasibility of LES for Wings and on the Hybrid RANS/LES Approach, Advances in DNS/LES, Proceedings of the First AFOSR International Conference on DNS/LES, 1997

${ }^{77}$ P.R. Spalart, S. Deck, M.L. Shur, K.D. Squires, M.Kh. Strelets, A.K. Travin, A.K. A new version of detached-eddy simulation, resistant to ambiguous grid densities. Theoretical and Computational Fluid Dynamics, vol. 20 (3), pp. 181-195, 2006

${ }^{78}$ M.L. Shur, P.R. Spalart, M.Kh. Strelets, A.K. Travin. A hybrid RANS-LES approach with delayed-DES and wall-modelled LES capabilities. Int. J. of Heat and Fluid Flow, vol. 29, pp. 1638-1649, 2008.

${ }^{79}$ F. Grossi, M. Braza, Y. Hoarau. Prediction of transonic buffet by delayed detached-eddy simulation, AIAA Journal 52 , pp. 2300-2312, 2014.

${ }^{80}$ C. Moussaed, S. Wornom, B. Koobus, A. Dervieux, T. Deloze, R. El Akoury, D. Szubert, Y. Hoarau, M. Braza. VMS and OES-based hybrid simulations of bluff body flows. Advances in Fluid-Structure Interaction, Notes on Numerical Fluid Mechanics and Multidisciplinary Design, Volume 133 : Updated contributions reflecting new findings presented at the ERCOFTAC Symposium on Unsteady Separation in Fluid-Structure Interaction, 17-21 June 2013, St John Hotel, Mykonos, Greece; by M. Braza, A. Bottaro, M. Thompson, 2016.

${ }^{81}$ J. Donea, S. Giuliani, J.P. Halleux. An arbitrary lagrangianeulerian finite element method for transient dynamic fluidstructure interactions, Comp. Methods in Applied Mechanics and Engineering, vol. 33 (1-3), pp. 689-723, 1982.

${ }^{82}$ D. Szubert, I. Asproulias, F. Grossi, R. Duvigneau, Y. Hoarau, M. Braza. Numerical study of the turbulent transonic interaction and transition location effect involving optimisation around a supercritical aerofoil, European Journal of MechanicsB/Fluids, to be published, 2016.

${ }^{83}$ D. Zilli. Numerical Simulation and modeling of morphing deformation on a supercritical airfoil wing at high Reynolds number, Master dissertation, ENSEEIHT-INPT, 2015

${ }^{84}$ D. Szubert, F. Grossi, A. Jimenez-Garcia, Y. Hoarau, J. Hunt, M. Braza. Shock-vortex shear-layer interaction in the transonic flow around a supercritical airfoil at high Reynolds number in buffet conditions. J. Fluids and Structures, vol. 55, pp. $276302,2015$.

${ }^{85}$ J.C.R. Hunt, I. Eames, J. Westerweel. Vortical interactions with interfacial shear layers. In: Kaneda, Y. (Ed.), IUTAM Symposium on Computational Physics and New Perspectives in Turbulence. Vol. 4 of IUTAM Bookseries. Springer, Nagoya, Japan, pp. 331338, 2008.

${ }^{86}$ D. Szubert. Physics and modelling of unsteady turbulent flows around aerodynamic and hydrodynamic structures at high Reynolds number by numerical simulation, INPT PhD thesis, 2015.

${ }^{87}$ R.B. Langtry, F.R. Menter. Correlation-Based Transition Modeling for Unstructured Parallelized Computational Fluid Dynamics Codes, AIAA Journal, vol. 47 (12), 2009.

${ }^{88}$ M. Elhimer, G. Harran, Y. Hoarau, S. Cazin, M. Marchal, M. Braza. Coherent and turbulent processes in the bistable regime around a tandem of cylinders including reattached flow dynamics by means of high-speed PIV, J. Fluids and Structures, in print, 2015.

${ }^{89}$ L. Jenkins, M. Khorrami, M. Choudhari, C. McGinley. Characterization of Unsteady Flow Structures Around Tandem Cylinders for Component Interaction Studies in Airframe Noise, AIAA paper 2005-2812, 2005

${ }^{90}$ D. Szubert, I. Jang, I. Park, M. Braza. Numerical simulations of oblique shock - boundary layer interaction at a high Reynolds number, Edition of the Center for Turbulence Research - CTR, http://ctr.stanford.edu/publications.html, https://ctr.stanford.edu/proceedings-2014-summer-program, Stanford University, USA, September 2014.

${ }^{91}$ Hasanzadeh, K., Laurendeau, E., Paraschivoiu, I., 2013. Quasi-steady con- vergence of Multistep NavierStokes Icing Simulations. Journal of Aircraft 50 (4), 12611274.

${ }^{92}$ Beaugendre, H., Morency, F., Habashi, W. G., 2006. Development of a sec- ond generation in-flight icing simulation code. Journal of fluids engineering 128 (2), 378387.

${ }^{93}$ Emmanuel Montreuil, O., Arnaud Chazottes, O., Didier Guffond, O., Angelo Murrone, O., Francois Caminade, D.-A., Stephane Catris, E., 2009. ECLIPPS: 1. three-dimensional CFD prediction of the ice accretion.

${ }^{94}$ Bourgault, Y., Habashi, W. G., Dompierre, J., Baruzzi, G. S., 1999. A finite element method study of Eulerian droplets impingement models. International Journal for Numerical Methods in Fluids 29 (4), 429449.

${ }^{95}$ Messinger B.L., Equilibrium Temperature of an Unheated Icing Surface as a Function of Airspeed, J. of Aeronautical Sciences Vol. 20(1), pp. 29-42, 1953.

${ }^{96}$ Kreith, F., Manglik, R., Bohn, M., 2010. Principles of heat transfer. Cengage learning. 
${ }^{97}$ Shin, J., Bond, T. H., 1992. Experimental and computational ice shapes and resulting drag increase for a NACA 0012 airfoil. National Aeronautics and Space Administration.

${ }^{98}$ Broeren, A. P., Bragg, M. B., Addy, H. E., Lee, S., Moens, F., Guffond, D., 2010. Effect of High-Fidelity Ice-Accretion Simulations on Full-Scale Airfoil performance. Journal of aircraft 47 (1), 240254.

${ }^{99} \mathrm{~W}$. Wright. Users manual for the improved NASA Lewis ice accretion code LEWICE 1.6, 1996

${ }^{100}$ LEWICE validation CD-ROM.

${ }^{101}$ Jung, S., Myong, R., Jan. 2013. Numerical modeling for eulerian droplet impingement in supercooled large droplet conditions. American Institute of Aeronautics and Astronautics.

${ }^{102}$ Sang, W., Shi, Y., Xi, C., Sep. 2013. Numerical simulation of icing effect and ice accretion on three-dimensional configurations. Science China Techno- logical Sciences 56 (9), 22782288

${ }^{103}$ T. Deloze, E. Laurendeau. NSMB contribution to the 2nd High Lift Prediction Workshop. AIAA Scitech, Jan. 2014, National Harbor, Maryland.

${ }^{104}$ R. Rudnik, K. Huber, S. Melber-Wilkending. EUROLIFT Test Case Description for the 2nd High Lift Prediction Workshop. AIAA Paper 2012-2924, June 2012.

${ }^{105}$ T. Pulliam, A. Sclafani. High Lift OVERFLOW Analysis of the DLR F11 Wind Tunnel Model. 32nd AIAA Applied Aerodynamics Conference, June 2014.

${ }^{106}$ Park C., Assessment of Two-Temperature Kinetic Model for Ionizing Air, Journal of Thermophysics and Heat Transfer, Vol. 3, No. 3, 1989.

${ }^{107}$ Park C., Review of Chemical Kinetic Problems of Future NASA Missions, I: Earth Entries, Journal of Thermophysics and Heat Transfer, Vol. 7, No. 3, 1993.

${ }^{108}$ Park C., Chemical-Kinetic Parameters of Hyperbolic Earth Entry, Journal of Thermophysics and Heat Transfer, Vol. 15, No. 1, 2001.

${ }^{109}$ Hanson, R.K. and Salimian S., Survey of Rate Constants in the N/H/O System, Combustion Chemistry, Edited by Gardiner, W.C. Springer Verlag, 1984

${ }^{110}$ Dunn M.G. and Kang S.W., Theoretical and Experimental Studise of Reentry Plasmas, NASA CR-2232, 1973.

${ }^{111}$ Srinivasan, S., Tannehill, J.C. and Weilmnster, K.J., Simplified Curve Fits for the Thermodynamic Properties of Equilibrium Air, NASA Report 1988

${ }^{112}$ Oppelstrup, J., Implementation of Real Gas Effects in a Finite Volume Navier Stokes Solver, Report TRITA-NA-P9002, KTH-Stockholm, January 1990

${ }^{113}$ Blottner, F.G., Johnson, M. and Ellis, M., Chemically Reacting Viscous Flow Program for Multi Component Gas, Mixture Sandia Laboratories, SC-RR-70-754, 1970

${ }^{114}$ Gupta, R.N and Yos J.M., A Review of Reaction Rates and Thermodyanmic and Transport Properties for an 11-Species Air Model for Chemical and Thermal Nonequilibrium Calculations to 30'000 K, NASA R-1232, 1990.

${ }^{115}$ Aupoix B. and Spalart P.R., Extensions of the Spalart-Allmaras turbulence model to account for wall roughness, Int. Journal of Heat and Fluid Flow, Vol. 24, pp. 454-462, 2003.

${ }^{116}$ Knopp T., Eisfeld B., Calvo J.B., A new extension for k-w turbulence models to account for wall roughness, Int. Journal of Heat and Fluid Flow, Vol. 30, pp. 54-65, 2009.

${ }^{117}$ Aupoix B., A general Strategy to Extend Turbulence Models to Rough Surfaces: Application to Smiths k-L model, Journal of Fluids Engineering, Vol. 129, pp. 1245-1254, 2007.

${ }^{118}$ Kemp N.H.,m Rose P.H., Detra R.W., Laminar Heat Transfer Around Blunt Bodies in Dissociated Air, AVCO Research Laboratory, Research Report 15, 1955.

${ }^{119}$ Vaglio-Laurin R., Turbulent Heat Transfer on Blunt-Nosed Bodies in Two-Dimensional and General Three-Dimensional Hypersonic Flow, Journal of the AeroSpace Sciences, Vol. 27, No. 1, 1960.

${ }^{120}$ Summary of Experimental and Analytical Results, Interim Report: Passive Nosetip Technology (PANT) Program, Vol. X, SAMSO_TR_74-86, 1975.

${ }^{121}$ Scoggins J.B. and Magin T.E., Development of Mutation++: Multicomponent Thermodynamic and Transport Properties for Ionized Plasmas written in C++, AIAA Paper 2014-2966, 11th AIAA/ASME Joint Thermophysics and Heat Transfer Conference, 2014. 